\title{
Spin-State Energetics and Spin-Crossover Behavior of Pseudotetrahedral Cobalt(III)-Imido Complexes. The Critical Role of the Tripodal Supporting Ligand
}

\section{(Supporting Information)}

Ingar H. Wasbotten and Abhik Ghosh*

Center for Theoretical and Computational Chemistry, Department of Chemistry, University of Tromsø, N-9037 Tromsø, Norway; abhik@chem.uit.no

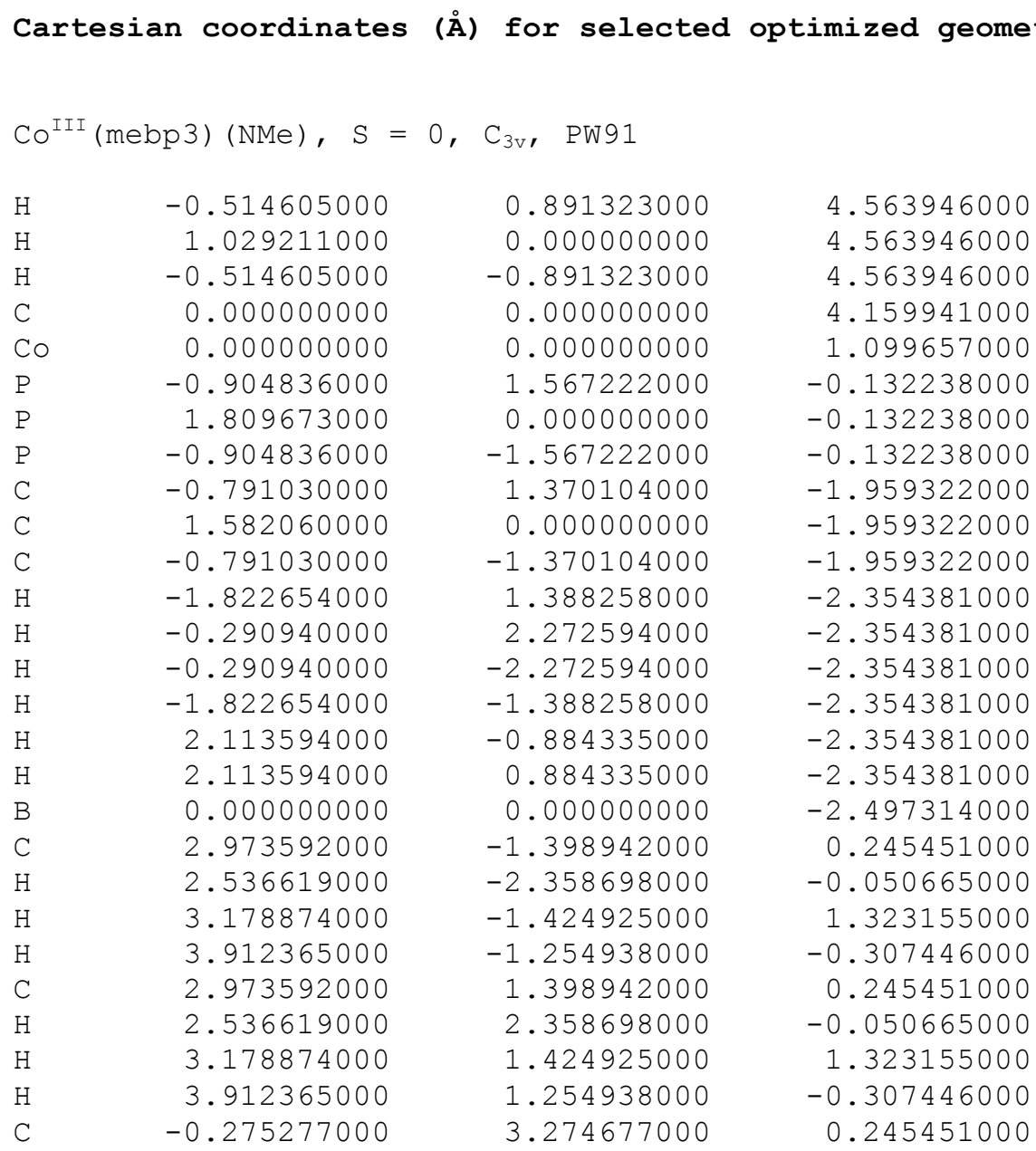




$\begin{array}{lr}\mathrm{H} & 0.774383000 \\ \mathrm{H} & -0.355415000 \\ \mathrm{H} & -0.869374000 \\ \mathrm{C} & -2.698315000 \\ \mathrm{H} & -3.311002000 \\ \mathrm{H} & -2.823458000 \\ \mathrm{H} & -3.042991000 \\ \mathrm{C} & -2.698315000 \\ \mathrm{H} & -3.311002000 \\ \mathrm{H} & -2.823458000 \\ \mathrm{H} & -3.042991000 \\ \mathrm{C} & -0.275277000 \\ \mathrm{H} & 0.774383000 \\ \mathrm{H} & -0.355415000 \\ \mathrm{H} & -0.869374000 \\ \mathrm{~N} & 0.000000000 \\ \mathrm{C} & 0.000000000 \\ \mathrm{H} & -0.508865000 \\ \mathrm{H} & -0.508865000 \\ \mathrm{H} & 1.017731000 \\ & \end{array}$

$$
\begin{array}{r}
3.376126000 \\
3.465448000 \\
4.015677000 \\
1.875735000 \\
1.017428000 \\
2.040523000 \\
2.760739000 \\
-1.875735000 \\
-1.017428000 \\
-2.040523000 \\
-2.760739000 \\
-3.274677000 \\
-3.376126000 \\
-3.465448000 \\
-4.015677000 \\
0.000000000 \\
0.000000000 \\
0.881381000 \\
-0.881381000 \\
0.000000000
\end{array}
$$

-0.050665000
1.323155000
-0.307446000
0.245451000
-0.050665000
1.323155000
-0.307446000
0.245451000
-0.050665000
1.323155000
-0.307446000
0.245451000
-0.050665000
1.323155000
-0.307446000
2.743600000
-4.149277000
-4.575221000
-4.575221000
-4.575221000
$\mathrm{Co}^{\text {III }}(\operatorname{mebp} 3)(\mathrm{NMe}), \mathrm{S}=0, \mathrm{C}_{3 \mathrm{v}}, \mathrm{OLYP}$

$\begin{array}{lr}\mathrm{H} & -0.513436000 \\ \mathrm{H} & 1.026872000 \\ \mathrm{H} & -0.513436000 \\ \mathrm{C} & 0.000000000 \\ \mathrm{CO} & 0.000000000 \\ \mathrm{P} & -0.911395000 \\ \mathrm{P} & 1.822790000 \\ \mathrm{P} & -0.911395000 \\ \mathrm{C} & -0.794128000 \\ \mathrm{C} & 1.588256000 \\ \mathrm{C} & -0.794128000 \\ \mathrm{H} & -1.822469000 \\ \mathrm{H} & -0.298052000 \\ \mathrm{H} & -0.298052000 \\ \mathrm{H} & -1.822469000 \\ \mathrm{H} & 2.120521000 \\ \mathrm{H} & 2.120521000 \\ \mathrm{~B} & 0.000000000 \\ \mathrm{C} & 2.993147000 \\ \mathrm{H} & 2.574893000 \\ \mathrm{H} & 3.189888000 \\ \mathrm{H} & 3.936116000 \\ \mathrm{C} & 2.993147000 \\ \mathrm{H} & 2.574893000 \\ \mathrm{H} & 3.189888000 \\ \mathrm{H} & 3.936116000 \\ \mathrm{C} & -0.286726000 \\ \mathrm{H} & 0.753470000 \\ \mathrm{H} & -0.355681000 \\ \mathrm{H} & -0.890285000 \\ \mathrm{C} & -2.706421000 \\ \mathrm{H} & -3.328363000 \\ \mathrm{H} & -2.834207000 \\ & \end{array}$

0.889298000

0.000000000

$-0.889298000$

0.000000000

0.000000000

1.578582000

0.000000000

$-1.578582000$

1. 375470000

0.000000000

$-1.375470000$

1.396364000

2. 276487000

$-2.276487000$

$-1.396364000$

$-0.880123000$

0.880123000

0.000000000

$-1.397012000$

$-2.356648000$

$-1.430978000$

$-1.244505000$

1. 397012000

2. 356648000

1. 430978000

1. 244505000

3.290647000

3. 408247000

3. 478012000

4.031029000

1.893635000

1.051599000

2. 047035000
4.564528000

4.564528000

4.564528000

4.158290000

1.093555000

$-0.131504000$

$-0.131504000$

$-0.131504000$

$-1.958832000$

$-1.958832000$

$-1.958832000$

$-2.353694000$

$-2.353694000$

$-2.353694000$

$-2.353694000$

$-2.353694000$

$-2.353694000$

$-2.497303000$

0.250494000

$-0.056628000$

1.326951000

$-0.287512000$

0.250494000

$-0.056628000$

1.326951000

$-0.287512000$

0.250494000

$-0.056628000$

1. 326951000

$-0.287512000$

0.250494000

$-0.056628000$

1.326951000 


$\begin{array}{lrrr}\mathrm{H} & -3.045831000 & 2.786523000 & -0.287512000 \\ \mathrm{C} & -2.706421000 & -1.893635000 & 0.250494000 \\ \mathrm{H} & -3.328363000 & -1.051599000 & -0.056628000 \\ \mathrm{H} & -2.834207000 & -2.047035000 & 1.326951000 \\ \mathrm{H} & -3.045831000 & -2.786523000 & -0.287512000 \\ \mathrm{C} & -0.286726000 & -3.290647000 & 0.250494000 \\ \mathrm{H} & 0.753470000 & -3.408247000 & -0.056628000 \\ \mathrm{H} & -0.355681000 & -3.478012000 & 1.326951000 \\ \mathrm{H} & -0.890285000 & -4.031029000 & -0.287512000 \\ \mathrm{~N} & 0.000000000 & 0.000000000 & 2.741122000 \\ \mathrm{C} & 0.000000000 & 0.000000000 & -4.158517000 \\ \mathrm{H} & -0.507009000 & 0.878166000 & -4.587596000 \\ \mathrm{H} & -0.507009000 & -0.878166000 & -4.587596000 \\ \mathrm{H} & 1.014018000 & 0.000000000 & -4.587596000\end{array}$

$\mathrm{Co}^{\text {III }}$ (mebp3) (NMe), $\mathrm{S}=0, \mathrm{C}_{1}, \mathrm{PW} 91$

$\begin{array}{lrrr}\mathrm{H} & -0.479207000 & 0.931654000 & 4.553041000 \\ \mathrm{H} & 1.052808000 & 0.020066000 & 4.557830000 \\ \mathrm{H} & -0.502629000 & -0.850771000 & 4.567922000 \\ \mathrm{C} & 0.023057000 & 0.030317000 & 4.156413000 \\ \mathrm{Co} & 0.018762000 & 0.013713000 & 1.096231000 \\ \mathrm{P} & -0.882715000 & 1.587122000 & -0.130495000 \\ \mathrm{P} & 1.825602000 & 0.007778000 & -0.139119000 \\ \mathrm{P} & -0.896184000 & -1.550003000 & -0.137312000 \\ \mathrm{C} & -0.773137000 & 1.392596000 & -1.958158000 \\ \mathrm{C} & 1.593205000 & 0.016509000 & -1.965710000 \\ \mathrm{C} & -0.786344000 & -1.347832000 & -1.963719000 \\ \mathrm{H} & -1.804498000 & 1.419551000 & -2.352738000 \\ \mathrm{H} & -0.266658000 & 2.292299000 & -2.351595000 \\ \mathrm{H} & -0.294131000 & -2.251609000 & -2.365138000 \\ \mathrm{H} & -1.819169000 & -1.356740000 & -2.355970000 \\ \mathrm{H} & 2.124798000 & -0.864552000 & -2.367385000 \\ \mathrm{H} & 2.121962000 & 0.903858000 & -2.357940000 \\ \mathrm{~B} & 0.009970000 & 0.020024000 & -2.499825000 \\ \mathrm{C} & 2.987878000 & -1.395997000 & 0.225910000 \\ \mathrm{H} & 2.549417000 & -2.353079000 & -0.076334000 \\ \mathrm{H} & 3.196952000 & -1.430224000 & 1.302658000 \\ \mathrm{H} & 3.925189000 & -1.249108000 & -0.328574000 \\ \mathrm{C} & 2.994027000 & 1.401236000 & 0.244537000 \\ \mathrm{H} & 2.560238000 & 2.363469000 & -0.047841000 \\ \mathrm{H} & 3.197735000 & 1.422219000 & 1.322581000 \\ \mathrm{H} & 3.933381000 & 1.256957000 & -0.307289000 \\ \mathrm{C} & -0.248200000 & 3.292658000 & 0.247636000 \\ \mathrm{H} & 0.798997000 & 3.394469000 & -0.056799000 \\ \mathrm{H} & -0.319768000 & 3.481222000 & 1.326239000 \\ \mathrm{H} & -0.845730000 & 4.036114000 & -0.298453000 \\ \mathrm{C} & -2.673693000 & 1.898535000 & 0.254490000 \\ \mathrm{H} & -3.289281000 & 1.042764000 & -0.042842000 \\ \mathrm{H} & -2.793794000 & 2.058044000 & 1.333485000 \\ \mathrm{H} & -3.019104000 & 2.787200000 & -0.291959000 \\ \mathrm{C} & -2.689837000 & -1.854790000 & 0.242680000 \\ \mathrm{H} & -3.303297000 & -1.002816000 & -0.069722000 \\ \mathrm{H} & -2.817473000 & -2.002090000 & 1.322559000 \\ \mathrm{H} & -3.032399000 & -2.749450000 & -0.295808000 \\ \mathrm{C} & -0.270998000 & -3.259433000 & 0.238616000\end{array}$




$\begin{array}{rrrr}\mathrm{H} & 0.780809000 & -3.361047000 & -0.049364000 \\ \mathrm{H} & -0.359157000 & -3.452600000 & 1.315174000 \\ \mathrm{H} & -0.861614000 & -3.998990000 & -0.319756000 \\ \mathrm{~N} & 0.020380000 & 0.018157000 & 2.740002000 \\ \mathrm{C} & 0.006807000 & 0.020550000 & -4.151359000 \\ \mathrm{H} & -0.490664000 & 0.908793000 & -4.577035000 \\ \mathrm{H} & -0.515363000 & -0.854151000 & -4.575827000 \\ \mathrm{H} & 1.023777000 & 0.005756000 & -4.579386000\end{array}$

$\mathrm{Co}^{\text {III }}$ (mebp3) (NMe), $\mathrm{S}=0, \mathrm{C}_{1}, \mathrm{OLYP}$

$\begin{array}{lrrr}\mathrm{H} & -0.476114000 & 0.928943000 & 4.554979000 \\ \mathrm{H} & 1.053276000 & 0.021097000 & 4.558036000 \\ \mathrm{H} & -0.497625000 & -0.849359000 & 4.570088000 \\ \mathrm{C} & 0.025315000 & 0.030121000 & 4.154620000 \\ \mathrm{Co} & 0.018297000 & 0.013504000 & 1.089601000 \\ \mathrm{P} & -0.891677000 & 1.597390000 & -0.130421000 \\ \mathrm{P} & 1.839308000 & 0.008854000 & -0.139389000 \\ \mathrm{P} & -0.901134000 & -1.561142000 & -0.137010000 \\ \mathrm{C} & -0.780904000 & 1.395835000 & -1.957891000 \\ \mathrm{C} & 1.600263000 & 0.017745000 & -1.965772000 \\ \mathrm{C} & -0.784063000 & -1.356057000 & -1.963722000 \\ \mathrm{H} & -1.810371000 & 1.418729000 & -2.349679000 \\ \mathrm{H} & -0.284587000 & 2.296435000 & -2.353270000 \\ \mathrm{H} & -0.288997000 & -2.256838000 & -2.360406000 \\ \mathrm{H} & -1.812530000 & -1.375374000 & -2.358241000 \\ \mathrm{H} & 2.131249000 & -0.860695000 & -2.365965000 \\ \mathrm{H} & 2.131814000 & 0.899435000 & -2.358121000 \\ \mathrm{~B} & 0.010538000 & 0.020141000 & -2.500430000 \\ \mathrm{C} & 3.004058000 & -1.395123000 & 0.234489000 \\ \mathrm{H} & 2.585390000 & -2.350310000 & -0.085748000 \\ \mathrm{H} & 3.194383000 & -1.441273000 & 1.311644000 \\ \mathrm{H} & 3.950768000 & -1.239455000 & -0.295961000 \\ \mathrm{C} & 3.015663000 & 1.399126000 & 0.249184000 \\ \mathrm{H} & 2.602274000 & 2.361725000 & -0.055228000 \\ \mathrm{H} & 3.210049000 & 1.428437000 & 1.326195000 \\ \mathrm{H} & 3.959340000 & 1.244482000 & -0.286934000 \\ \mathrm{C} & -0.258341000 & 3.305784000 & 0.253955000 \\ \mathrm{H} & 0.778604000 & 3.422326000 & -0.064429000 \\ \mathrm{H} & -0.314439000 & 3.486762000 & 1.332242000 \\ \mathrm{H} & -0.865860000 & 4.051258000 & -0.272372000 \\ \mathrm{C} & -2.683814000 & 1.917690000 & 0.260479000 \\ \mathrm{H} & -3.310247000 & 1.080183000 & -0.049818000 \\ \mathrm{H} & -2.806603000 & 2.064046000 & 1.338482000 \\ \mathrm{H} & -3.021935000 & 2.815687000 & -0.269720000 \\ \mathrm{C} & -2.697325000 & -1.867697000 & 0.247409000 \\ \mathrm{H} & -3.317155000 & -1.028282000 & -0.070997000 \\ \mathrm{H} & -2.826178000 & -2.007186000 & 1.325624000 \\ \mathrm{H} & -3.038732000 & -2.766564000 & -0.279202000 \\ \mathrm{C} & -0.283809000 & -3.275638000 & 0.246186000 \\ \mathrm{H} & 0.755908000 & -3.398032000 & -0.060607000 \\ \mathrm{H} & -0.353298000 & -3.461030000 & 1.322953000 \\ \mathrm{H} & -0.890646000 & -4.014436000 & -0.290266000 \\ \mathrm{~N} & 0.021150000 & 0.017843000 & 2.737506000 \\ \mathrm{C} & 0.006848000 & 0.023588000 & -4.161005000 \\ \mathrm{H} & -0.462787000 & 0.923703000 & -4.587281000\end{array}$




$\begin{array}{lrrr}\mathrm{H} & -0.538598000 & -0.831047000 & -4.590623000 \\ \mathrm{H} & 1.018979000 & -0.019324000 & -4.592541000\end{array}$

\begin{tabular}{|c|c|c|c|}
\hline $\mathrm{Co}$ & 0.256053000 & 3.716541000 & 5.438258000 \\
\hline $\mathrm{N}$ & -1.739649000 & 4.781126000 & 3.587655000 \\
\hline $\mathrm{N}$ & 1.144396000 & 3.205938000 & 4.124997000 \\
\hline $\mathrm{N}$ & -0.059857000 & 1.096357000 & 6.713994000 \\
\hline $\mathrm{N}$ & 1.877005000 & 5.663194000 & 6.836428000 \\
\hline $\mathrm{N}$ & -0.207978000 & 2.579430000 & 8.276027000 \\
\hline $\mathrm{C}$ & -1.481475000 & 4.101989000 & 4.772512000 \\
\hline $\mathrm{C}$ & -0.039057000 & 2.471081000 & 6.904824000 \\
\hline $\mathrm{C}$ & 1.506576000 & 2.770316000 & 2.889836000 \\
\hline $\mathrm{N}$ & -0.145982000 & 6.425645000 & 6.841759000 \\
\hline $\mathrm{N}$ & -2.745146000 & 3.740136000 & 5.199190000 \\
\hline $\mathrm{C}$ & 4.501512000 & 3.034079000 & 7.175586000 \\
\hline $\mathrm{C}$ & -0.799438000 & 5.391367000 & 2.686402000 \\
\hline $\mathrm{C}$ & 5.445000000 & 3.484920000 & 6.250393000 \\
\hline $\mathrm{C}$ & -0.884293000 & 5.074492000 & 1.328876000 \\
\hline $\mathrm{C}$ & 0.585123000 & 5.311359000 & 6.469361000 \\
\hline $\mathrm{C}$ & 1.026077000 & 1.613981000 & 0.800903000 \\
\hline $\mathrm{C}$ & 3.310228000 & 3.735125000 & 7.364126000 \\
\hline $\mathrm{C}$ & -0.313231000 & 3.795317000 & 9.077805000 \\
\hline $\mathrm{H}$ & 0.252573000 & 4.585273000 & 8.583618000 \\
\hline $\mathrm{H}$ & 0.165752000 & 3.588027000 & 10.044851000 \\
\hline $\mathrm{C}$ & 3.066833000 & 4.885765000 & 6.615515000 \\
\hline $\mathrm{N}$ & -2.464921000 & 4.770900000 & 8.178052000 \\
\hline C & -3.730054000 & 4.179852000 & 4.321222000 \\
\hline $\mathrm{H}$ & -4.782100000 & 3.989958000 & 4.489808000 \\
\hline $\mathrm{C}$ & 0.662113000 & 7.406262000 & 7.411834000 \\
\hline $\mathrm{H}$ & 0.267250000 & 8.351484000 & 7.761424000 \\
\hline $\mathrm{C}$ & -0.041875000 & 5.708000000 & 0.415985000 \\
\hline $\mathrm{C}$ & 2.820021000 & 3.030988000 & 2.416685000 \\
\hline $\mathrm{C}$ & -3.110715000 & 3.046040000 & 6.430609000 \\
\hline $\mathrm{H}$ & -2.270207000 & 2.419903000 & 6.730073000 \\
\hline $\mathrm{H}$ & -3.959501000 & 2.389407000 & 6.193703000 \\
\hline $\mathrm{C}$ & 2.321699000 & 1.881468000 & 0.345444000 \\
\hline $\mathrm{C}$ & -3.102488000 & 4.826520000 & 3.311958000 \\
\hline $\mathrm{H}$ & -3.491685000 & 5.326339000 & 2.436021000 \\
\hline $\mathrm{C}$ & 4.003442000 & 5.343480000 & 5.687565000 \\
\hline $\mathrm{C}$ & 1.930860000 & 6.932063000 & 7.400777000 \\
\hline $\mathrm{H}$ & 2.859008000 & 7.369811000 & 7.743506000 \\
\hline $\mathrm{C}$ & 0.615852000 & 2.048704000 & 2.054589000 \\
\hline $\mathrm{C}$ & 1.431322000 & 0.055845000 & 5.074622000 \\
\hline C & -1.586693000 & 6.629961000 & 6.700669000 \\
\hline $\mathrm{H}$ & -1.921771000 & 6.102089000 & 5.806785000 \\
\hline $\mathrm{H}$ & -1.744878000 & 7.704010000 & 6.533472000 \\
\hline C & 3.213202000 & 2.589693000 & 1.159983000 \\
\hline $\mathrm{C}$ & 0.135232000 & 0.352236000 & 5.493202000 \\
\hline $\mathrm{C}$ & 0.520798000 & -1.358409000 & 3.336009000 \\
\hline $\mathrm{C}$ & 5.194473000 & 4.640635000 & 5.507894000 \\
\hline C & -0.348605000 & 1.335808000 & 8.882871000 \\
\hline $\mathrm{H}$ & -0.482566000 & 1.222610000 & 9.950967000 \\
\hline $\mathrm{C}$ & 0.119342000 & 6.339647000 & 3.135020000 \\
\hline
\end{tabular}




\begin{tabular}{|c|c|c|c|}
\hline $\mathrm{C}$ & -3.521727000 & 3.978377000 & 7.586922000 \\
\hline $\mathrm{H}$ & -4.317404000 & 4.651914000 & 7.229812000 \\
\hline $\mathrm{H}$ & -3.979537000 & 3.332080000 & 8.356848000 \\
\hline c & 0.886990000 & 6.649853000 & 0.858309000 \\
\hline c & -0.773684000 & -1.048775000 & 3.755862000 \\
\hline c & 0.964912000 & 6.961379000 & 2.217371000 \\
\hline c & -0.970980000 & -0.191813000 & 4.840465000 \\
\hline c & -2.420352000 & 6.192989000 & 7.917262000 \\
\hline $\mathrm{H}$ & -2.035920000 & 6.707919000 & 8.81197600 \\
\hline $\mathrm{H}$ & -3.441615000 & 6.578886000 & 7.74682100 \\
\hline c & -0.250977000 & 0.406333000 & 7.90409400 \\
\hline $\mathrm{F}$ & -0.275611000 & -0.674382000 & 7.93973900 \\
\hline c & 1.620122000 & -0.800478000 & 3.99095200 \\
\hline c & -1.759494000 & 4.255440000 & 9.33079600 \\
\hline $\mathrm{H}$ & -2.327836000 & 3.411941000 & 9.75418700 \\
\hline $\mathrm{H}$ & -1.709024000 & 5.027797000 & 10.11894200 \\
\hline $\mathrm{H}$ & 2.577159000 & 3.398726000 & $8.0942050 c$ \\
\hline $\mathrm{F}$ & 4.698913000 & 2.140650000 & 7.76637600 \\
\hline $\mathrm{H}$ & 6.378530000 & 2.941058000 & 6.11392500 \\
\hline $\mathrm{H}$ & 5.927875000 & 4.998698000 & 4.78695700 \\
\hline & 3.789255000 & 6.239236000 & 5.10601200 \\
\hline $\mathrm{F}$ & 3.503005000 & 3.578597000 & 3.06501100 \\
\hline $\mathrm{H}$ & 4.223160000 & 2.797075000 & 0.80656400 \\
\hline $\mathrm{F}$ & 2.637793000 & 1.536352000 & -0.63826400 \\
\hline $\mathrm{I}$ & 0.333586000 & 1.056022000 & 0.17003900 \\
\hline $\mathrm{F}$ & -0.385979000 & 1.837145000 & 2.42746400 \\
\hline $\mathrm{H}$ & 2.278428000 & 0.494440000 & 5.59940900 \\
\hline $\mathrm{H}$ & 2.630925000 & -1.035841000 & $3.6616660 c$ \\
\hline $\mathrm{H}$ & 0.674657000 & -2.035752000 & 2.49694500 \\
\hline $\mathrm{H}$ & -1.633658000 & -1.483494000 & 3.24822200 \\
\hline $\mathrm{H}$ & -1.975895000 & 0.039886000 & 5.19213500 \\
\hline $\mathrm{H}$ & -1.598120000 & 4.323005000 & 0.99344600 \\
\hline $\mathrm{H}$ & -0.107029000 & 5.453739000 & -0.64059100 \\
\hline $\mathrm{H}$ & 1.547083000 & 7.141984000 & 0.14525200 \\
\hline $\mathrm{H}$ & 1.679711000 & 7.705379000 & 2.56647600 \\
\hline . & 0.167984000 & 6.588448000 & 4.19174500 \\
\hline \multicolumn{4}{|c|}{$\mathrm{CO}^{\mathrm{III}}$ (timen) (NPh), $\mathrm{S}=0, \mathrm{C}_{1}, \mathrm{OLYP}, \mathrm{q}=+1$} \\
\hline $\mathrm{Co}$ & 0.220517000 & 3.705050000 & 5.40788200 \\
\hline $\mathrm{N}$ & -1.764733000 & 4.848184000 & 3.54199800 \\
\hline $\mathrm{N}$ & 1.101803000 & 3.176954000 & 4.08880000 \\
\hline $\mathrm{N}$ & -0.012468000 & 1.043233000 & 6.74240200 \\
\hline $\mathrm{N}$ & 1.911143000 & 5.648999000 & 6.81057300 \\
\hline $\mathrm{N}$ & -0.221969000 & 2.541457000 & 8.28102200 \\
\hline C & -1.512726000 & 4.154423000 & 4.72358500 \\
\hline $\mathrm{C}$ & -0.059060000 & 2.422652000 & 6.90694700 \\
\hline $\mathrm{C}$ & 1.483385000 & 2.744277000 & 2.85811000 \\
\hline $\mathrm{N}$ & -0.110273000 & 6.415661000 & 6.87087100 \\
\hline $\mathrm{N}$ & -2.782462000 & 3.799549000 & 5.14358300 \\
\hline C & 4.592289000 & 3.079131000 & 7.22609600 \\
\hline $\mathrm{C}$ & -0.837430000 & 5.495373000 & 2.64461700 \\
\hline $\mathrm{C}$ & 5.542718000 & 3.538041000 & 6.31260200 \\
\hline $\mathrm{C}$ & -0.908823000 & 5.191659000 & 1.28171200 \\
\hline $\mathrm{C}$ & 0.609979000 & 5.302931000 & 6.46325500 \\
\hline $\mathrm{C}$ & 1.062377000 & 1.599545000 & 0.74565300 \\
\hline
\end{tabular}




\begin{tabular}{|c|c|c|c|}
\hline $\mathrm{C}$ & 3.380081000 & 3.752193000 & 7.380272000 \\
\hline $\mathrm{C}$ & -0.371268000 & 3.756430000 & 9.081543000 \\
\hline $\mathrm{H}$ & 0.192980000 & 4.553023000 & 8.610872000 \\
\hline H & 0.092550000 & 3.553933000 & 10.053837000 \\
\hline & 3.115305000 & 4.881844000 & 6.605220000 \\
\hline$\sqrt{ }$ & -2.531448000 & 4.784413000 & 8.209382000 \\
\hline & -3.758887000 & 4.241196000 & 4.261437000 \\
\hline & -4.811058000 & 4.053254000 & 4.416685000 \\
\hline & 0.711796000 & 7.386796000 & 7.430075000 \\
\hline & 0.332985000 & 8.329564000 & 7.796660000 \\
\hline & -0.091127000 & 5.863854000 & 0.374102000 \\
\hline & 2.813385000 & 2.995468000 & 2.419739000 \\
\hline & -3.159096000 & 3.110078000 & 6.376108000 \\
\hline & -2.332459000 & 2.472893000 & 6.669368000 \\
\hline & -4.012691000 & 2.465083000 & 6.135890000 \\
\hline & 2.372958000 & 1.858509000 & 0.328875000 \\
\hline & -3.125519000 & 4.893346000 & 3.262213000 \\
\hline & -3.515942000 & 5.395840000 & 2.391776000 \\
\hline & 4.061584000 & 5.346605000 & 5.689153000 \\
\hline & 1.976378000 & 6.910458000 & 7.387811000 \\
\hline $\mathrm{H}$ & 2.907297000 & 7.346363000 & 7.716354000 \\
\hline & 0.616981000 & 2.031017000 & 1.987926000 \\
\hline C & 1.453738000 & -0.115235000 & 5.153482000 \\
\hline $\mathrm{C}$ & -1.552661000 & 6.642100000 & 6.756035000 \\
\hline $\mathrm{H}$ & -1.903121000 & 6.152507000 & 5.854616000 \\
\hline $\mathrm{H}$ & -1.694273000 & 7.719946000 & 6.617555000 \\
\hline C & 3.242732000 & 2.556946000 & 1.174057000 \\
\hline C & 0.166766000 & 0.256199000 & 5.542229000 \\
\hline C & 0.507864000 & -1.556145000 & 3.455177000 \\
\hline $\mathrm{C}$ & 5.275545000 & 4.674345000 & 5.547235000 \\
\hline $\mathrm{C}$ & -0.278032000 & 1.305274000 & 8.909477000 \\
\hline $\mathrm{H}$ & -0.391929000 & 1.198953000 & 9.978406000 \\
\hline $\mathrm{C}$ & 0.044689000 & 6.479264000 & 3.093720000 \\
\hline C & -3.572699000 & 4.025130000 & 7.554470000 \\
\hline . & -4.353554000 & 4.715059000 & 7.207925000 \\
\hline $\mathrm{H}$ & -4.057009000 & 3.359516000 & 8.286514000 \\
\hline C & 0.804512000 & 6.835267000 & 0.820683000 \\
\hline C & -0.776800000 & -1.178180000 & 3.848379000 \\
\hline $\mathrm{C}$ & 0.868059000 & 7.138256000 & 2.181331000 \\
\hline C & -0.951551000 & -0.272563000 & 4.895734000 \\
\hline $\mathrm{C}$ & -2.412132000 & 6.203014000 & 7.961588000 \\
\hline $\mathrm{H}$ & -2.028180000 & 6.690587000 & 8.866890000 \\
\hline $\mathrm{H}$ & -3.409801000 & 6.635837000 & 7.781286000 \\
\hline C & -0.142920000 & 0.367302000 & 7.946452000 \\
\hline $\mathrm{H}$ & -0.113005000 & -0.710009000 & 8.005076000 \\
\hline C & 1.620231000 & -1.019904000 & 4.105359000 \\
\hline $\mathrm{C}$ & -1.825205000 & 4.211202000 & 9.331416000 \\
\hline $\mathrm{H}$ & -2.399519000 & 3.362029000 & 9.723236000 \\
\hline $\mathrm{H}$ & -1.769956000 & 4.946890000 & 10.150250000 \\
\hline $\mathrm{H}$ & 2.651497000 & 3.407489000 & 8.105434000 \\
\hline $\mathrm{H}$ & 4.798428000 & 2.201842000 & 7.834899000 \\
\hline $\mathrm{H}$ & 6.490599000 & 3.016212000 & 6.202771000 \\
\hline 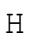 & 6.011568000 & 5.040479000 & 4.835542000 \\
\hline $\mathrm{H}$ & 3.843817000 & 6.225751000 & 5.088121000 \\
\hline $\mathrm{H}$ & 3.483169000 & 3.533780000 & 3.082727000 \\
\hline 1 & 4.263619000 & 2.759687000 & 0.855232000 \\
\hline & 2.714843000 & 1.516654000 & -0.645564000 \\
\hline
\end{tabular}




$\begin{array}{rrrr}\mathrm{H} & 0.385003000 & 1.051992000 & 0.092630000 \\ \mathrm{H} & -0.396993000 & 1.824656000 & 2.316414000 \\ \mathrm{H} & 2.314234000 & 0.298663000 & 5.670889000 \\ \mathrm{H} & 2.623378000 & -1.306140000 & 3.799631000 \\ \mathrm{H} & 0.642641000 & -2.266706000 & 2.642973000 \\ \mathrm{H} & -1.647703000 & -1.595541000 & 3.348191000 \\ \mathrm{H} & -1.950392000 & 0.005119000 & 5.221827000 \\ \mathrm{H} & -1.590709000 & 4.421319000 & 0.932547000 \\ \mathrm{H} & -0.148055000 & 5.614259000 & -0.682526000 \\ \mathrm{H} & 1.447560000 & 7.353233000 & 0.112910000 \\ \mathrm{H} & 1.554190000 & 7.902881000 & 2.539415000 \\ \mathrm{H} & 0.087472000 & 6.734661000 & 4.143122000\end{array}$

$\begin{array}{lrrr}\mathrm{CO}^{\mathrm{III}}\left(\mathrm{Tp}^{\mathrm{tBu}}, \mathrm{Me}\right)\left(\mathrm{N}^{t} \mathrm{Bu}\right), \mathrm{S}=\mathrm{N}, \mathrm{C}_{3 \mathrm{v}}, \mathrm{PW} 91 & \\ \mathrm{Co} & 0.000000000 & 0.000000000 & -0.011881000 \\ \mathrm{~N} & 0.717709000 & -1.243108000 & 2.542544000 \\ \mathrm{~N} & 0.828202000 & -1.434488000 & 1.190111000 \\ \mathrm{~N} & 0.717709000 & 1.243108000 & 2.542544000 \\ \mathrm{~N} & 0.828202000 & 1.434488000 & 1.190111000 \\ \mathrm{~N} & -1.435417000 & 0.000000000 & 2.542544000 \\ \mathrm{~N} & -1.656404000 & 0.000000000 & 1.190111000 \\ \mathrm{~N} & 0.000000000 & 0.000000000 & -1.668880000 \\ \mathrm{~B} & 0.000000000 & 0.000000000 & 3.070777000 \\ \mathrm{C} & 1.347006000 & -2.333083000 & 4.719484000 \\ \mathrm{C} & 1.302517000 & -2.256026000 & 3.226836000 \\ \mathrm{C} & 1.812304000 & -3.139002000 & 2.286726000 \\ \mathrm{C} & 1.502086000 & -2.601688000 & 1.023589000 \\ \mathrm{C} & 1.858965000 & -3.219822000 & -0.313156000 \\ \mathrm{C} & 0.569938000 & -3.531763000 & -1.094200000 \\ \mathrm{C} & 2.773627000 & -2.259462000 & -1.094200000 \\ \mathrm{C} & 2.620383000 & -4.538637000 & -0.096611000 \\ \mathrm{C} & 1.347006000 & 2.333083000 & 4.719484000 \\ \mathrm{C} & 1.302517000 & 2.256026000 & 3.226836000 \\ \mathrm{C} & 1.812304000 & 3.139002000 & 2.286726000 \\ \mathrm{C} & 1.502086000 & 2.601688000 & 1.023589000 \\ \mathrm{C} & 1.858965000 & 3.219822000 & -0.313156000 \\ \mathrm{C} & 2.620383000 & 4.538637000 & -0.096611000 \\ \mathrm{C} & 2.773627000 & 2.259462000 & -1.094200000 \\ \mathrm{C} & 0.569938000 & 3.531763000 & -1.094200000 \\ \mathrm{C} & -2.694012000 & 0.000000000 & 4.719484000 \\ \mathrm{C} & -2.605034000 & 0.000000000 & 3.226836000 \\ \mathrm{C} & -3.624607000 & 0.000000000 & 2.286726000 \\ \mathrm{C} & -3.004171000 & 0.000000000 & 1.023589000 \\ \mathrm{C} & -3.717930000 & 0.000000000 & -0.313156000 \\ \mathrm{C} & -5.240766000 & 0.000000000 & -0.096611000 \\ \mathrm{C} & -3.343565000 & 1.272300000 & -1.094200000 \\ \mathrm{C} & -3.343565000 & -1.272300000 & -1.094200000 \\ \mathrm{C} & 0.000000000 & 0.000000000 & -3.121790000 \\ \mathrm{C} & 1.446599000 & 0.000000000 & -3.665073000 \\ \mathrm{C} & -0.723300000 & -1.252792000 & -3.665073000 \\ \mathrm{C} & -0.723300000 & 1.252792000 & -3.665073000 \\ \mathrm{H} & 1.992739000 & 0.889427000 & -3.335743000 \\ \mathrm{H} & -0.226103000 & -2.170476000 & -3.335743000 \\ & & & \end{array}$




\begin{tabular}{|c|c|c|c|}
\hline $\mathrm{H}$ & -1.766636000 & 1.281049000 & -3.335743000 \\
\hline $\mathrm{H}$ & 0.000000000 & 0.000000000 & 4.267963000 \\
\hline $\mathrm{H}$ & 1.873797000 & -3.245512000 & 5.019438000 \\
\hline $\mathrm{H}$ & 1.873807000 & -1.475914000 & 5.159035000 \\
\hline $\mathrm{H}$ & 0.341276000 & -2.360722000 & 5.159035000 \\
\hline $\mathrm{H}$ & 2.343820000 & -4.059616000 & 2.494889000 \\
\hline $\mathrm{H}$ & 0.814081000 & -3.940101000 & -2.086240000 \\
\hline $\mathrm{H}$ & -0.037110000 & -2.629574000 & -1.219106000 \\
\hline $\mathrm{H}$ & -0.031584000 & -4.276318000 & -0.554731000 \\
\hline $\mathrm{H}$ & 2.295833000 & -1.282649000 & -1.219106000 \\
\hline $\mathrm{H}$ & 3.005187000 & -2.675065000 & -2.086240000 \\
\hline $\mathrm{H}$ & 3.719192000 & -2.110807000 & -0.554731000 \\
\hline $\mathrm{H}$ & 2.013318000 & -5.269672000 & 0.453118000 \\
\hline $\mathrm{H}$ & 2.872678000 & -4.975625000 & -1.072683000 \\
\hline $\mathrm{H}$ & 3.557011000 & -4.378421000 & 0.453118000 \\
\hline $\mathrm{H}$ & 0.341276000 & 2.360722000 & 5.159035000 \\
\hline $\mathrm{H}$ & 1.873807000 & 1.475914000 & 5.159035000 \\
\hline $\mathrm{H}$ & 1.873797000 & 3.245512000 & 5.019438000 \\
\hline $\mathrm{H}$ & 2.343820000 & 4.059616000 & 2.494889000 \\
\hline $\mathrm{H}$ & 2.872678000 & 4.975625000 & -1.072683000 \\
\hline $\mathrm{H}$ & 2.013318000 & 5.269672000 & 0.453118000 \\
\hline $\mathrm{H}$ & 3.557011000 & 4.378421000 & 0.453118000 \\
\hline $\mathrm{H}$ & 2.295833000 & 1.282649000 & -1.219106000 \\
\hline $\mathrm{H}$ & 3.005187000 & 2.675065000 & -2.086240000 \\
\hline $\mathrm{H}$ & 3.719192000 & 2.110807000 & -0.554731000 \\
\hline $\mathrm{H}$ & 0.814081000 & 3.940101000 & -2.086240000 \\
\hline $\mathrm{H}$ & -0.037110000 & 2.629574000 & -1.219106000 \\
\hline $\mathrm{H}$ & -0.031584000 & 4.276318000 & -0.554731000 \\
\hline $\mathrm{H}$ & -2.215083000 & -0.884807000 & 5.159035000 \\
\hline $\mathrm{H}$ & -3.747595000 & 0.000000000 & 5.019438000 \\
\hline $\mathrm{H}$ & -2.215083000 & 0.884807000 & 5.159035000 \\
\hline $\mathrm{H}$ & -4.687640000 & 0.000000000 & 2.494889000 \\
\hline $\mathrm{H}$ & -5.570329000 & 0.891251000 & 0.453118000 \\
\hline $\mathrm{H}$ & -5.570329000 & -0.891251000 & 0.453118000 \\
\hline $\mathrm{H}$ & -5.745357000 & 0.000000000 & -1.072683000 \\
\hline $\mathrm{H}$ & -2.258723000 & 1.346926000 & -1.219106000 \\
\hline $\mathrm{H}$ & -3.819268000 & 1.265035000 & -2.086240000 \\
\hline $\mathrm{H}$ & -3.687608000 & 2.165511000 & -0.554731000 \\
\hline $\mathrm{H}$ & -3.687608000 & -2.165511000 & -0.554731000 \\
\hline $\mathrm{H}$ & -3.819268000 & -1.265035000 & -2.086240000 \\
\hline $\mathrm{H}$ & -2.258723000 & -1.346926000 & -1.219106000 \\
\hline $\mathrm{H}$ & 1.413609000 & 0.000000000 & -4.763774000 \\
\hline $\mathrm{H}$ & 1.992739000 & -0.889427000 & -3.335743000 \\
\hline $\mathrm{H}$ & -1.766636000 & -1.281049000 & -3.335743000 \\
\hline $\mathrm{H}$ & -0.706805000 & -1.224222000 & -4.763774000 \\
\hline $\mathrm{H}$ & -0.706805000 & 1.224222000 & -4.763774000 \\
\hline $\mathrm{H}$ & -0.226103000 & 2.170476000 & -3.335743000 \\
\hline \multicolumn{4}{|c|}{$\mathrm{CO}^{\mathrm{III}}\left(\mathrm{Tp}^{t B u, M e}\right)\left(\mathrm{N}^{t} \mathrm{Bu}\right), \mathrm{S}=0, \mathrm{C}_{3 \mathrm{v}}, \mathrm{OLYP}$} \\
\hline $\mathrm{Co}$ & 0.000000000 & 0.000000000 & -0.089080000 \\
\hline $\mathrm{N}$ & 0.726385000 & -1.258136000 & 2.507665000 \\
\hline $\mathrm{N}$ & 0.859784000 & -1.489190000 & 1.169562000 \\
\hline $\mathrm{N}$ & 0.726385000 & 1.258136000 & 2.507665000 \\
\hline $\mathrm{N}$ & 0.859784000 & 1.489190000 & 1.169562000 \\
\hline $\mathrm{N}$ & -1.452770000 & 0.000000000 & 2.507665000 \\
\hline
\end{tabular}




\begin{tabular}{|c|c|c|c|}
\hline $\mathrm{N}$ & -1.719569000 & 0.000000000 & 1.169562000 \\
\hline $\mathrm{N}$ & 0.000000000 & 0.000000000 & -1.745431000 \\
\hline B & 0.000000000 & 0.000000000 & 3.009925000 \\
\hline $\mathrm{C}$ & 1.332931000 & -2.308704000 & 4.731180000 \\
\hline C & 1.298862000 & -2.249694000 & 3.234475000 \\
\hline $\mathrm{C}$ & 1.823561000 & -3.158500000 & 2.331432000 \\
\hline $\mathrm{C}$ & 1.536895000 & -2.661980000 & 1.047794000 \\
\hline C & 1.927059000 & -3.337764000 & -0.262707000 \\
\hline $\mathrm{C}$ & 0.655314000 & -3.693026000 & -1.063262000 \\
\hline $\mathrm{C}$ & 2.870598000 & -2.414032000 & -1.063262000 \\
\hline $\mathrm{C}$ & 2.686726000 & -4.653545000 & 0.015195000 \\
\hline $\mathrm{C}$ & 1.332931000 & 2.308704000 & 4.731180000 \\
\hline $\mathrm{C}$ & 1.298862000 & 2.249694000 & 3.234475000 \\
\hline $\mathrm{C}$ & 1.823561000 & 3.158500000 & 2.331432000 \\
\hline $\mathrm{C}$ & 1.536895000 & 2.661980000 & 1.047794000 \\
\hline $\mathrm{C}$ & 1.927059000 & 3.337764000 & -0.262707000 \\
\hline $\mathrm{C}$ & 2.686726000 & 4.653545000 & 0.015195000 \\
\hline $\mathrm{C}$ & 2.870598000 & 2.414032000 & -1.063262000 \\
\hline $\mathrm{C}$ & 0.655314000 & 3.693026000 & -1.063262000 \\
\hline $\mathrm{C}$ & -2.665861000 & 0.000000000 & 4.731180000 \\
\hline $\mathrm{C}$ & -2.597723000 & 0.000000000 & 3.234475000 \\
\hline C & -3.647122000 & 0.000000000 & 2.331432000 \\
\hline $\mathrm{C}$ & -3.073790000 & 0.000000000 & 1.047794000 \\
\hline $\mathrm{C}$ & -3.854118000 & 0.000000000 & -0.262707000 \\
\hline $\mathrm{C}$ & -5.373451000 & 0.000000000 & 0.015195000 \\
\hline $\mathrm{C}$ & -3.525912000 & 1.278994000 & -1.063262000 \\
\hline $\mathrm{C}$ & -3.525912000 & -1.278994000 & -1.063262000 \\
\hline $\mathrm{C}$ & 0.000000000 & 0.000000000 & -3.200290000 \\
\hline $\mathrm{C}$ & 1.452883000 & 0.000000000 & -3.745392000 \\
\hline $\mathrm{C}$ & -0.726441000 & -1.258234000 & -3.745392000 \\
\hline C & -0.726441000 & 1.258234000 & -3.745392000 \\
\hline $\mathrm{H}$ & 1.999655000 & 0.885678000 & -3.419368000 \\
\hline $\mathrm{H}$ & -0.232808000 & -2.174591000 & -3.419368000 \\
\hline $\mathrm{H}$ & -1.766847000 & 1.288913000 & -3.419368000 \\
\hline $\mathrm{H}$ & 0.000000000 & 0.000000000 & 4.200818000 \\
\hline $\mathrm{H}$ & 1.857118000 & -3.216623000 & 5.040971000 \\
\hline $\mathrm{H}$ & 1.858799000 & -1.453563000 & 5.168875000 \\
\hline $\mathrm{H}$ & 0.329423000 & -2.336548000 & 5.168875000 \\
\hline $\mathrm{H}$ & 2.348538000 & -4.067787000 & 2.584773000 \\
\hline $\mathrm{H}$ & 0.920329000 & -4.140441000 & -2.029345000 \\
\hline $\mathrm{H}$ & 0.039129000 & -2.813613000 & -1.241002000 \\
\hline $\mathrm{H}$ & 0.048726000 & -4.419062000 & -0.510059000 \\
\hline $\mathrm{H}$ & 2.417096000 & -1.440693000 & -1.241002000 \\
\hline $\mathrm{H}$ & 3.125562000 & -2.867249000 & -2.029345000 \\
\hline $\mathrm{H}$ & 3.802657000 & -2.251729000 & -0.510059000 \\
\hline $\mathrm{H}$ & 2.074806000 & -5.369166000 & 0.573390000 \\
\hline $\mathrm{H}$ & 2.957955000 & -5.123329000 & -0.936928000 \\
\hline $\mathrm{H}$ & 3.612431000 & -4.481417000 & 0.573390000 \\
\hline $\mathrm{H}$ & 0.329423000 & 2.336548000 & 5.168875000 \\
\hline $\mathrm{H}$ & 1.858799000 & 1.453563000 & 5.168875000 \\
\hline $\mathrm{H}$ & 1.857118000 & 3.216623000 & 5.040971000 \\
\hline $\mathrm{H}$ & 2.348538000 & 4.067787000 & 2.584773000 \\
\hline $\mathrm{H}$ & 2.957955000 & 5.123329000 & -0.936928000 \\
\hline $\mathrm{H}$ & 2.074806000 & 5.369166000 & 0.573390000 \\
\hline $\mathrm{H}$ & 3.612431000 & 4.481417000 & 0.573390000 \\
\hline $\mathrm{H}$ & 2.417096000 & 1.440693000 & -1.241002000 \\
\hline $\mathrm{H}$ & 3.125562000 & 2.867249000 & -2.029345000 \\
\hline
\end{tabular}




$\begin{array}{lr}\mathrm{H} & 3.802657000 \\ \mathrm{H} & 0.920329000 \\ \mathrm{H} & 0.039129000 \\ \mathrm{H} & 0.048726000 \\ \mathrm{H} & -2.188222000 \\ \mathrm{H} & -3.714236000 \\ \mathrm{H} & -2.188222000 \\ \mathrm{H} & -4.697076000 \\ \mathrm{H} & -5.687237000 \\ \mathrm{H} & -5.687237000 \\ \mathrm{H} & -5.915910000 \\ \mathrm{H} & -2.456225000 \\ \mathrm{H} & -4.045892000 \\ \mathrm{H} & -3.851383000 \\ \mathrm{H} & -3.851383000 \\ \mathrm{H} & -4.045892000 \\ \mathrm{H} & -2.456225000 \\ \mathrm{H} & 1.426458000 \\ \mathrm{H} & 1.999655000 \\ \mathrm{H} & -1.766847000 \\ \mathrm{H} & -0.713229000 \\ \mathrm{H} & -0.713229000 \\ \mathrm{H} & -0.232808000 \\ & \end{array}$

$\begin{array}{rr}2.251729000 & -0.510059000 \\ 4.140441000 & -2.029345000 \\ 2.813613000 & -1.241002000 \\ 4.419062000 & -0.510059000 \\ -0.882986000 & 5.168875000 \\ 0.000000000 & 5.040971000 \\ 0.882986000 & 5.168875000 \\ 0.000000000 & 2.584773000 \\ 0.887748000 & 0.573390000 \\ -0.887748000 & 0.573390000 \\ 0.000000000 & -0.936928000 \\ 1.372919000 & -1.241002000 \\ 1.273192000 & -2.029345000 \\ 2.167333000 & -0.510059000 \\ -2.167333000 & -0.510059000 \\ -1.273192000 & -2.029345000 \\ -1.372919000 & -1.241002000 \\ 0.000000000 & -4.841364000 \\ -0.885678000 & -3.419368000 \\ -1.288913000 & -3.419368000 \\ -1.235349000 & -4.841364000 \\ 1.235349000 & -4.841364000 \\ 2.174591000 & -3.419368000\end{array}$

\begin{tabular}{|c|c|c|c|}
\hline Co & -0.016088000 & -0.004070000 & 0.018958000 \\
\hline $\mathrm{N}$ & 0.699996000 & -1.249730000 & 2.573008000 \\
\hline N & 0.809598000 & -1.441430000 & 1.220871000 \\
\hline $\mathrm{N}$ & 0.700473000 & 1.236252000 & 2.576802000 \\
\hline $\mathrm{N}$ & 0.811309000 & 1.429441000 & 1.225148000 \\
\hline N & -1.454373000 & -0.005836000 & 2.574902000 \\
\hline $\mathrm{N}$ & -1.676861000 & -0.004968000 & 1.223422000 \\
\hline $\mathrm{N}$ & -0.015626000 & -0.003943000 & -1.636880000 \\
\hline B & -0.018715000 & -0.007182000 & 3.102566000 \\
\hline $\mathrm{C}$ & 1.336988000 & -2.337612000 & 4.749652000 \\
\hline C & 1.287139000 & -2.261910000 & 3.257189000 \\
\hline C & 1.796941000 & -3.144753000 & 2.316188000 \\
\hline $\mathrm{C}$ & 1.484493000 & -2.607771000 & 1.053532000 \\
\hline $\mathrm{C}$ & 1.841163000 & -3.225511000 & -0.283609000 \\
\hline C & 0.552843000 & -3.539964000 & -1.064882000 \\
\hline $\mathrm{C}$ & 2.752996000 & -2.264057000 & -1.066228000 \\
\hline C & 2.605293000 & -4.543050000 & -0.067335000 \\
\hline $\mathrm{C}$ & 1.333937000 & 2.320936000 & 4.756273000 \\
\hline $\mathrm{C}$ & 1.288157000 & 2.246817000 & 3.263407000 \\
\hline C & 1.800024000 & 3.130097000 & 2.324549000 \\
\hline C & 1.488198000 & 2.595725000 & 1.060445000 \\
\hline $\mathrm{C}$ & 1.845731000 & 3.214712000 & -0.275403000 \\
\hline C & 2.608440000 & 4.532710000 & -0.056852000 \\
\hline C & 2.759411000 & 2.254456000 & -1.057433000 \\
\hline $\mathrm{C}$ & 0.557479000 & 3.529085000 & -1.056667000 \\
\hline $\mathrm{C}$ & -2.711731000 & -0.008971000 & 4.753794000 \\
\hline C & -2.623714000 & -0.008920000 & 3.260755000 \\
\hline $\mathrm{C}$ & -3.644104000 & -0.010603000 & 2.321781000 \\
\hline C & -3.025156000 & -0.007541000 & 1.057801000 \\
\hline $\mathrm{C}$ & -3.740213000 & -0.004889000 & -0.278033000 \\
\hline
\end{tabular}




\begin{tabular}{|c|c|c|c|}
\hline $\mathrm{C}$ & -5.263076000 & -0.004181000 & -0.058742000 \\
\hline & -3.366078000 & 1.267868000 & -1.058351000 \\
\hline c & -3.368887000 & -1.275137000 & -1.063882000 \\
\hline 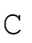 & -0.014895000 & -0.003676000 & -3.088309000 \\
\hline 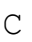 & 1.432283000 & -0.005185000 & -3.632455000 \\
\hline & -0.740270000 & -1.256035000 & -3.632970000 \\
\hline c & -0.737000000 & 1.250805000 & -3.63101800 \\
\hline F & 1.980179000 & 0.882705000 & -3.30154400 \\
\hline $\mathrm{H}$ & -0.243841000 & -2.174562000 & -3.304492000 \\
\hline $\mathrm{H}$ & -1.781861000 & 1.276768000 & -3.30626500 \\
\hline $\mathrm{F}$ & -0.018458000 & -0.009103000 & 4.29959500 \\
\hline $\mathrm{H}$ & 1.870628000 & -3.246050000 & 5.04855500 \\
\hline $\mathrm{H}$ & 1.859954000 & -1.476603000 & 5.18664500 \\
\hline F & 0.332993000 & -2.371287000 & 5.19336800 \\
\hline $\mathrm{F}$ & 2.328258000 & -4.065103000 & 2.524346000 \\
\hline $\mathrm{H}$ & 0.798796000 & -3.950338000 & -2.05555700 \\
\hline H & -0.054816000 & -2.638434000 & -1.19305800 \\
\hline $\mathrm{H}$ & -0.048889000 & -4.283839000 & -0.52485400 \\
\hline $\mathrm{H}$ & 2.273959000 & -1.287852000 & -1.190197000 \\
\hline H & 2.982118000 & -2.679681000 & -2.058988000 \\
\hline $\mathrm{H}$ & 3.699896000 & -2.114462000 & -0.52953200 \\
\hline $\mathrm{H}$ & 1.999625000 & -5.275499000 & 0.48210500 \\
\hline $\mathrm{H}$ & 2.859217000 & -4.979097000 & -1.04286700 \\
\hline $\mathrm{F}$ & 3.541207000 & -4.380654000 & 0.48328600 \\
\hline F & 0.328671000 & 2.359193000 & 5.196733000 \\
\hline $\mathrm{H}$ & 1.851348000 & 1.457184000 & 5.19443500 \\
\hline $\mathrm{H}$ & 1.870808000 & 3.226728000 & 5.05754600 \\
\hline $\mathrm{H}$ & 2.334053000 & 4.048472000 & 2.53437800 \\
\hline $\mathrm{H}$ & 2.859939000 & 4.971827000 & -1.03173200 \\
\hline $\mathrm{H}$ & 2.002452000 & 5.262714000 & 0.49564900 \\
\hline $\mathrm{H}$ & 3.545517000 & 4.370426000 & 0.491773000 \\
\hline $\mathrm{H}$ & 2.282941000 & 1.276866000 & -1.18050400 \\
\hline $\mathrm{H}$ & 2.987804000 & 2.669631000 & -2.05065000 \\
\hline $\mathrm{H}$ & 3.706744000 & 2.107764000 & -0.52055700 \\
\hline $\mathrm{H}$ & 0.803272000 & 3.936770000 & -2.04860900 \\
\hline $\mathrm{H}$ & -0.051838000 & 2.628305000 & -1.182264000 \\
\hline $\mathrm{H}$ & -0.042639000 & 4.275143000 & -0.517848000 \\
\hline $\mathrm{H}$ & -2.230913000 & -0.892808000 & 5.19360500 \\
\hline $\mathrm{H}$ & -3.764745000 & -0.010641000 & 5.05448600 \\
\hline $\mathrm{H}$ & -2.233989000 & 0.876923000 & 5.19294900 \\
\hline $\mathrm{H}$ & -4.706837000 & -0.011790000 & 2.53131200 \\
\hline $\mathrm{H}$ & -5.590543000 & 0.885602000 & 0.49493100 \\
\hline $\mathrm{H}$ & -5.592095000 & -0.896778000 & 0.489196000 \\
\hline $\mathrm{H}$ & -5.769854000 & -0.000634000 & -1.03319300 \\
\hline $\mathrm{H}$ & -2.281278000 & 1.343407000 & -1.18305200 \\
\hline $\mathrm{H}$ & -3.841403000 & 1.259684000 & -2.05076700 \\
\hline $\mathrm{H}$ & -3.711345000 & 2.161025000 & -0.519689000 \\
\hline $\mathrm{H}$ & -3.713217000 & -2.170104000 & -0.527607000 \\
\hline $\mathrm{H}$ & -3.847012000 & -1.263068000 & -2.05471900 \\
\hline $\mathrm{H}$ & -2.284383000 & -1.350900000 & -1.19208200 \\
\hline $\mathrm{H}$ & 1.398011000 & -0.002995000 & -4.73104800 \\
\hline $\mathrm{H}$ & 1.976943000 & -0.896470000 & -3.30546600 \\
\hline H & -1.783515000 & -1.283097000 & -3.30314500 \\
\hline $\mathrm{H}$ & -0.723802000 & -1.226125000 & -4.731396000 \\
\hline 11 & -0.715174000 & 1.225447000 & -4.72966500 \\
\hline & -0.241772000 & 2.167626000 & -3.29622000 \\
\hline
\end{tabular}




\begin{tabular}{|c|c|c|c|}
\hline $\mathrm{CO}$ & -0.016100000 & -0.004100000 & 0.019000000 \\
\hline $\mathrm{N}$ & 0.700000000 & -1.249700000 & 2.573000000 \\
\hline $\mathrm{N}$ & 0.809600000 & -1.441400000 & 1.220900000 \\
\hline $\mathrm{N}$ & 0.700500000 & 1.236300000 & 2.576800000 \\
\hline $\mathrm{N}$ & 0.811300000 & 1.429400000 & 1.225100000 \\
\hline $\mathrm{N}$ & -1.454400000 & -0.005800000 & 2.574900000 \\
\hline $\mathrm{N}$ & -1.676900000 & -0.005000000 & 1.223400000 \\
\hline $\mathrm{N}$ & -0.015600000 & -0.003900000 & -1.636900000 \\
\hline B & -0.018700000 & -0.007200000 & 3.102600000 \\
\hline $\mathrm{C}$ & 1.337000000 & -2.337600000 & 4.749700000 \\
\hline $\mathrm{C}$ & 1.287100000 & -2.261900000 & 3.257200000 \\
\hline $\mathrm{C}$ & 1.796900000 & -3.144800000 & 2.316200000 \\
\hline C & 1.484500000 & -2.607800000 & 1.053500000 \\
\hline $\mathrm{C}$ & 1.841200000 & -3.225500000 & -0.283600000 \\
\hline $\mathrm{C}$ & 0.552800000 & -3.540000000 & -1.064900000 \\
\hline $\mathrm{C}$ & 2.753000000 & -2.264100000 & -1.066200000 \\
\hline $\mathrm{C}$ & 2.605300000 & -4.543100000 & -0.067300000 \\
\hline $\mathrm{C}$ & 1.333900000 & 2.320900000 & 4.756300000 \\
\hline $\mathrm{C}$ & 1.288200000 & 2.246800000 & 3.263400000 \\
\hline $\mathrm{C}$ & 1.800000000 & 3.130100000 & 2.324500000 \\
\hline $\mathrm{C}$ & 1.488200000 & 2.595700000 & 1.060400000 \\
\hline C & 1.845700000 & 3.214700000 & -0.275400000 \\
\hline $\mathrm{C}$ & 2.608400000 & 4.532700000 & -0.056900000 \\
\hline $\mathrm{C}$ & 2.759400000 & 2.254500000 & -1.057400000 \\
\hline C & 0.557500000 & 3.529100000 & -1.056700000 \\
\hline $\mathrm{C}$ & -2.711700000 & -0.009000000 & 4.753800000 \\
\hline $\mathrm{C}$ & -2.623700000 & -0.008900000 & 3.260800000 \\
\hline $\mathrm{C}$ & -3.644100000 & -0.010600000 & 2.321800000 \\
\hline $\mathrm{C}$ & -3.025200000 & -0.007500000 & 1.057800000 \\
\hline $\mathrm{C}$ & -3.740200000 & -0.004900000 & -0.278000000 \\
\hline $\mathrm{C}$ & -5.263100000 & -0.004200000 & -0.058700000 \\
\hline $\mathrm{C}$ & -3.366100000 & 1.267900000 & -1.058400000 \\
\hline $\mathrm{C}$ & -3.368900000 & -1.275100000 & -1.063900000 \\
\hline C & -0.014900000 & -0.003700000 & -3.088300000 \\
\hline $\mathrm{C}$ & 1.432300000 & -0.005200000 & -3.632500000 \\
\hline $\mathrm{C}$ & -0.740300000 & -1.256000000 & -3.633000000 \\
\hline $\mathrm{C}$ & -0.737000000 & 1.250800000 & -3.631000000 \\
\hline $\mathrm{H}$ & 1.980200000 & 0.882700000 & -3.301500000 \\
\hline $\mathrm{H}$ & -0.243800000 & -2.174600000 & -3.304500000 \\
\hline $\mathrm{H}$ & -1.781900000 & 1.276800000 & -3.306300000 \\
\hline $\mathrm{H}$ & -0.018500000 & -0.009100000 & 4.299600000 \\
\hline $\mathrm{H}$ & 1.870600000 & -3.246000000 & 5.048600000 \\
\hline $\mathrm{H}$ & 1.860000000 & -1.476600000 & 5.186600000 \\
\hline $\mathrm{H}$ & 0.333000000 & -2.371300000 & 5.193400000 \\
\hline $\mathrm{H}$ & 2.328300000 & -4.065100000 & 2.524300000 \\
\hline $\mathrm{H}$ & 0.798800000 & -3.950300000 & -2.055600000 \\
\hline $\mathrm{H}$ & -0.054800000 & -2.638400000 & -1.193100000 \\
\hline $\mathrm{H}$ & -0.048900000 & -4.283800000 & -0.524900000 \\
\hline $\mathrm{H}$ & 2.274000000 & -1.287900000 & -1.190200000 \\
\hline $\mathrm{H}$ & 2.982100000 & -2.679700000 & -2.059000000 \\
\hline $\mathrm{H}$ & 3.699900000 & -2.114500000 & -0.529500000 \\
\hline $\mathrm{H}$ & 1.999600000 & -5.275500000 & 0.482100000 \\
\hline $\mathrm{H}$ & 2.859200000 & -4.979100000 & -1.042900000 \\
\hline $\mathrm{H}$ & 3.541200000 & -4.380700000 & 0.483300000 \\
\hline
\end{tabular}




\begin{tabular}{|c|c|c|c|}
\hline $\mathrm{H}$ & 0.328700000 & 2.359200000 & 5.196700000 \\
\hline $\mathrm{H}$ & 1.851300000 & 1.457200000 & 5.194400000 \\
\hline $\mathrm{H}$ & 1.870800000 & 3.226700000 & 5.057500000 \\
\hline $\mathrm{H}$ & 2.334100000 & 4.048500000 & 2.534400000 \\
\hline $\mathrm{H}$ & 2.859900000 & 4.971800000 & -1.031700000 \\
\hline $\mathrm{H}$ & 2.002500000 & 5.262700000 & 0.495600000 \\
\hline $\mathrm{H}$ & 3.545500000 & 4.370400000 & 0.491800000 \\
\hline $\mathrm{H}$ & 2.282900000 & 1.276900000 & -1.180500000 \\
\hline $\mathrm{H}$ & 2.987800000 & 2.669600000 & -2.050600000 \\
\hline $\mathrm{H}$ & 3.706700000 & 2.107800000 & -0.520600000 \\
\hline $\mathrm{H}$ & 0.803300000 & 3.936800000 & -2.048600000 \\
\hline $\mathrm{H}$ & -0.051800000 & 2.628300000 & -1.182300000 \\
\hline $\mathrm{H}$ & -0.042600000 & 4.275100000 & -0.517800000 \\
\hline $\mathrm{H}$ & -2.230900000 & -0.892800000 & 5.193600000 \\
\hline $\mathrm{H}$ & -3.764700000 & -0.010600000 & 5.054500000 \\
\hline $\mathrm{H}$ & -2.234000000 & 0.876900000 & 5.192900000 \\
\hline $\mathrm{H}$ & -4.706800000 & -0.011800000 & 2.531300000 \\
\hline $\mathrm{H}$ & -5.590500000 & 0.885600000 & 0.494900000 \\
\hline $\mathrm{H}$ & -5.592100000 & -0.896800000 & 0.489200000 \\
\hline $\mathrm{H}$ & -5.769900000 & -0.000600000 & -1.033200000 \\
\hline $\mathrm{H}$ & -2.281300000 & 1.343400000 & -1.183100000 \\
\hline $\mathrm{H}$ & -3.841400000 & 1.259700000 & -2.050800000 \\
\hline $\mathrm{H}$ & -3.711300000 & 2.161000000 & -0.519700000 \\
\hline $\mathrm{H}$ & -3.713200000 & -2.170100000 & -0.527600000 \\
\hline $\mathrm{H}$ & -3.847000000 & -1.263100000 & -2.054700000 \\
\hline $\mathrm{H}$ & -2.284400000 & -1.350900000 & -1.192100000 \\
\hline $\mathrm{H}$ & 1.398000000 & -0.003000000 & -4.731000000 \\
\hline $\mathrm{H}$ & 1.976900000 & -0.896500000 & -3.305500000 \\
\hline $\mathrm{H}$ & -1.783500000 & -1.283100000 & -3.303100000 \\
\hline $\mathrm{H}$ & -0.723800000 & -1.226100000 & -4.731400000 \\
\hline $\mathrm{H}$ & -0.715200000 & 1.225400000 & -4.729700000 \\
\hline $\mathrm{H}$ & -0.241800000 & 2.167600000 & -3.296200000 \\
\hline \multicolumn{4}{|c|}{$\mathrm{CO}^{\mathrm{III}}\left(\mathrm{Tp}^{t \mathrm{Bu}, \mathrm{Me}}\right)\left(\mathrm{N}^{t} \mathrm{Bu}\right), \mathrm{S}=1, \mathrm{C}_{1}, \mathrm{PW} 91$} \\
\hline $\mathrm{Co}$ & 0.184510000 & -0.000519000 & -0.049189000 \\
\hline $\mathrm{N}$ & 0.734430000 & -1.250069000 & 2.526872000 \\
\hline $\mathrm{N}$ & 0.897030000 & -1.455361000 & 1.181597000 \\
\hline $\mathrm{N}$ & 0.732446000 & 1.248200000 & 2.527433000 \\
\hline $\mathrm{N}$ & 0.893188000 & 1.455391000 & 1.182167000 \\
\hline $\mathrm{N}$ & -1.449876000 & -0.002875000 & 2.512726000 \\
\hline $\mathrm{N}$ & -1.716546000 & -0.001288000 & 1.165909000 \\
\hline $\mathrm{N}$ & -0.172535000 & -0.000349000 & -1.720585000 \\
\hline B & -0.006409000 & -0.001856000 & 3.031049000 \\
\hline C & 1.235460000 & -2.361159000 & 4.726779000 \\
\hline C & 1.257034000 & -2.281041000 & 3.233976000 \\
\hline $\mathrm{C}$ & 1.781160000 & -3.184196000 & 2.317220000 \\
\hline $\mathrm{C}$ & 1.538596000 & -2.642851000 & 1.043439000 \\
\hline $\mathrm{C}$ & 1.906498000 & -3.262741000 & -0.289242000 \\
\hline C & 0.621462000 & -3.582250000 & -1.076437000 \\
\hline C & 2.807404000 & -2.298528000 & -1.081196000 \\
\hline C & 2.678366000 & -4.573423000 & -0.061104000 \\
\hline C & 1.232611000 & 2.358751000 & 4.727945000 \\
\hline $\mathrm{C}$ & 1.252198000 & 2.280253000 & 3.235007000 \\
\hline $\mathrm{C}$ & 1.772225000 & 3.186374000 & 2.318372000 \\
\hline C & 1.530247000 & 2.645375000 & 1.044434000 \\
\hline
\end{tabular}




\begin{tabular}{|c|c|c|c|}
\hline C & 1.892219000 & 3.268755000 & -0.288238000 \\
\hline 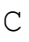 & 2.662977000 & 4.580154000 & -0.060567000 \\
\hline $\mathrm{C}$ & 2.791154000 & 2.308009000 & -1.086526000 \\
\hline$c$ & 0.603030000 & 3.588169000 & -1.068732000 \\
\hline C & -2.654319000 & -0.008695000 & 4.729378000 \\
\hline c & -2.601858000 & -0.005170000 & 3.23438000 \\
\hline$c$ & -3.650629000 & -0.004611000 & 2.327141000 \\
\hline$c$ & -3.062803000 & -0.002063000 & 1.046853000 \\
\hline$c$ & -3.795284000 & -0.000337000 & -0.280383000 \\
\hline c & -5.314624000 & -0.001258000 & -0.04319500 \\
\hline$c$ & -3.422985000 & 1.267205000 & -1.07091000 \\
\hline c & -3.422249000 & -1.265267000 & -1.07461000 \\
\hline$c$ & -0.072782000 & 0.000552000 & -3.15615400 \\
\hline C & 1.385812000 & -0.006939000 & -3.669399000 \\
\hline C & -0.795928000 & -1.249112000 & -3.71895000 \\
\hline C & -0.782340000 & 1.259290000 & -3.71590400 \\
\hline $\mathrm{H}$ & 1.927802000 & 0.878843000 & -3.32154300 \\
\hline $\mathrm{H}$ & -0.322434000 & -2.169456000 & -3.35967800 \\
\hline $\mathrm{H}$ & -1.835278000 & 1.280726000 & -3.415719000 \\
\hline $\mathrm{H}$ & -0.009859000 & -0.002058000 & 4.22800900 \\
\hline $\mathrm{H}$ & 1.741851000 & -3.277318000 & 5.04854700 \\
\hline $\mathrm{H}$ & 1.747364000 & -1.507338000 & 5.18905100 \\
\hline $\mathrm{H}$ & 0.210832000 & -2.381882000 & 5.12021700 \\
\hline $\mathrm{F}$ & 2.278636000 & -4.117409000 & 2.55160800 \\
\hline $\mathrm{H}$ & 0.873719000 & -4.001239000 & -2.061992000 \\
\hline $\mathrm{H}$ & 0.016774000 & -2.679249000 & -1.219101000 \\
\hline $\mathrm{H}$ & 0.012862000 & -4.318992000 & -0.53444000 \\
\hline H & 2.301350000 & -1.340287000 & -1.24728100 \\
\hline $\mathrm{H}$ & 3.061757000 & -2.732367000 & -2.05945500 \\
\hline $\mathrm{H}$ & 3.741019000 & -2.103560000 & -0.53602200 \\
\hline $\mathrm{H}$ & 2.072107000 & -5.305855000 & 0.487770000 \\
\hline $\mathrm{H}$ & 2.942574000 & -5.015346000 & -1.031214000 \\
\hline $\mathrm{H}$ & 3.607735000 & -4.402023000 & 0.49789300 \\
\hline $\mathrm{H}$ & 0.208552000 & 2.376067000 & 5.12301300 \\
\hline $\mathrm{H}$ & 1.747797000 & 1.505902000 & 5.18841300 \\
\hline $\mathrm{H}$ & 1.736800000 & 3.275939000 & 5.04997700 \\
\hline $\mathrm{H}$ & 2.267041000 & 4.120857000 & 2.553194000 \\
\hline $\mathrm{H}$ & 2.922643000 & 5.024301000 & -1.03088900 \\
\hline $\mathrm{H}$ & 2.057783000 & 5.310660000 & 0.49202400 \\
\hline $\mathrm{H}$ & 3.594804000 & 4.409062000 & 0.49441000 \\
\hline $\mathrm{H}$ & 2.286382000 & 1.348865000 & -1.25127600 \\
\hline $\mathrm{H}$ & 3.039125000 & 2.743949000 & -2.065485000 \\
\hline $\mathrm{H}$ & 3.728202000 & 2.114439000 & -0.546753000 \\
\hline $\mathrm{H}$ & 0.849961000 & 4.009588000 & -2.05458300 \\
\hline $\mathrm{H}$ & -0.001231000 & 2.684739000 & -1.21038000 \\
\hline $\mathrm{H}$ & -0.003868000 & 4.322985000 & -0.52222700 \\
\hline $\mathrm{H}$ & -2.164587000 & -0.893583000 & 5.15694900 \\
\hline $\mathrm{H}$ & -3.700425000 & -0.011458000 & 5.053870000 \\
\hline $\mathrm{H}$ & -2.167729000 & 0.875813000 & 5.161322000 \\
\hline $\mathrm{H}$ & -4.706700000 & -0.006183000 & 2.57020000 \\
\hline $\mathrm{H}$ & -5.635809000 & 0.888505000 & 0.51441500 \\
\hline $\mathrm{H}$ & -5.635114000 & -0.893290000 & 0.51119400 \\
\hline H & -5.835497000 & 0.000246000 & -1.01056500 \\
\hline $\mathrm{H}$ & -2.341206000 & 1.313702000 & -1.233089000 \\
\hline $\mathrm{H}$ & -3.930233000 & 1.267956000 & -2.047817000 \\
\hline 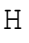 & -3.732105000 & 2.167952000 & -0.52259100 \\
\hline $\mathrm{H}$ & -3.730095000 & -2.167803000 & -0.52850300 \\
\hline
\end{tabular}


$-1.263835000$

$-1.310213000$

$-0.004932000$

$-0.900289000$

$-1.257911000$

$-1.234224000$

1.244336000

2.173534000
$-2.050923000$

$-1.237627000$

$-4.769381000$

$-3.324836000$

$-3.422019000$

$-4.816494000$

$-4.813496000$

$-3.357448000$
$\mathrm{CO}^{\text {III }}\left(\mathrm{Tp}^{t \mathrm{Bu}, \mathrm{Me}}\right)\left(\mathrm{N}^{t} \mathrm{Bu}\right), \mathrm{S}=1, \mathrm{C}_{1}, \mathrm{OLYP}$

\begin{tabular}{|c|c|c|}
\hline $\mathrm{Co}$ & 0.203135000 & -0.000163000 \\
\hline $\mathrm{N}$ & 0.713087000 & -1.264226000 \\
\hline $\mathrm{N}$ & 0.919713000 & -1.497793000 \\
\hline $\mathrm{N}$ & 0.710324000 & 1.262444000 \\
\hline $\mathrm{N}$ & 0.914094000 & 1.499046000 \\
\hline $\mathrm{N}$ & -1.504823000 & -0.003065000 \\
\hline $\mathrm{N}$ & -1.832186000 & -0.001908000 \\
\hline $\mathrm{N}$ & -0.121356000 & -0.001118000 \\
\hline B & -0.035388000 & -0.001984000 \\
\hline C & 1.187040000 & -2.344459000 \\
\hline C & 1.230794000 & -2.270343000 \\
\hline C & 1.792745000 & -3.184564000 \\
\hline C & 1.583440000 & -2.679667000 \\
\hline $\mathrm{C}$ & 2.014215000 & -3.349417000 \\
\hline C & 0.764127000 & -3.725527000 \\
\hline C & 2.952687000 & -2.415126000 \\
\hline C & 2.791341000 & -4.649431000 \\
\hline C & 1.184893000 & 2.339972000 \\
\hline C & 1.226245000 & 2.268797000 \\
\hline C & 1.783821000 & 3.186372000 \\
\hline C & 1.573686000 & 2.683291000 \\
\hline C & 1.997401000 & 3.358283000 \\
\hline C & 2.777929000 & 4.656019000 \\
\hline C & 2.929281000 & 2.426848000 \\
\hline C & 0.742272000 & 3.739791000 \\
\hline C & -2.639378000 & -0.006286000 \\
\hline $\mathrm{C}$ & -2.623348000 & -0.004430000 \\
\hline $\mathrm{C}$ & -3.710082000 & -0.004090000 \\
\hline C & -3.182006000 & -0.002387000 \\
\hline C & -3.982420000 & -0.001216000 \\
\hline C & -5.496073000 & 0.000901000 \\
\hline $\mathrm{C}$ & -3.653845000 & 1.270952000 \\
\hline C & -3.657600000 & -1.274392000 \\
\hline C & -0.040539000 & -0.002127000 \\
\hline $\mathrm{C}$ & 1.414402000 & -0.014854000 \\
\hline C & -0.784634000 & -1.253401000 \\
\hline C & -0.762290000 & 1.262668000 \\
\hline $\mathrm{H}$ & 1.967201000 & 0.864436000 \\
\hline $\mathrm{H}$ & -0.310412000 & -2.175035000 \\
\hline $\mathrm{H}$ & -1.803891000 & 1.292043000 \\
\hline $\mathrm{H}$ & -0.015826000 & -0.002590000 \\
\hline $\mathrm{H}$ & 1.696533000 & -3.254468000 \\
\hline $\mathrm{H}$ & 1.687184000 & -1.49216300 \\
\hline $\mathrm{H}$ & 0.162269000 & -2.37847500 \\
\hline $\mathrm{H}$ & 2.292106000 & -4.1006740 \\
\hline
\end{tabular}

$-0.143184000$

2. 477936000

1.147331000

2. 479202000

1. 148688000

2. 504987000

1.178753000

$-1.828471000$

2.967939000

4.721170000

3. 225442000

2. 347560000

1.054636000

$-0.246925000$

$-1.073719000$

$-1.041438000$

0.054128000

4.723649000

3. 227677000

2.350510000

1.056987000

$-0.244209000$

0.057948000

$-1.049475000$

$-1.061126000$

4. 780260000

3. 281317000

2. 423327000

1.117659000

$-0.181617000$

0.119337000

$-0.994231000$

$-0.994180000$

$-3.263487000$

$-3.805946000$

$-3.812936000$

$-3.811776000$

$-3.469022000$

$-3.469824000$

$-3.486820000$

4.157843000

5.048568000

5.192575000

5.104686000

2.626859000 


\begin{tabular}{|c|c|c|c|}
\hline $\mathrm{H}$ & 1.057186000 & -4.179185000 & -2.028643000 \\
\hline $\mathrm{H}$ & 0.144026000 & -2.853535000 & -1.277918000 \\
\hline $\mathrm{H}$ & 0.150061000 & -4.451072000 & -0.528487000 \\
\hline $\mathrm{H}$ & 2.470366000 & -1.467149000 & -1.277609000 \\
\hline $\mathrm{H}$ & 3.256787000 & -2.888567000 & -1.983041000 \\
\hline $\mathrm{H}$ & 3.858037000 & -2.196835000 & -0.463969000 \\
\hline $\mathrm{H}$ & 2.178002000 & -5.373985000 & 0.598858000 \\
\hline $\mathrm{H}$ & 3.095608000 & -5.118499000 & -0.888077000 \\
\hline $\mathrm{H}$ & 3.698041000 & -4.457890000 & 0.636959000 \\
\hline $\mathrm{H}$ & 0.160711000 & 2.370750000 & 5.109003000 \\
\hline $\mathrm{H}$ & 1.687960000 & 1.488023000 & 5.192549000 \\
\hline $\mathrm{H}$ & 1.692709000 & 3.250630000 & 5.051901000 \\
\hline $\mathrm{H}$ & 2.280802000 & 4.103527000 & 2.630639000 \\
\hline $\mathrm{H}$ & 3.078401000 & 5.128177000 & -0.883928000 \\
\hline $\mathrm{H}$ & 2.168333000 & 5.379520000 & 0.608238000 \\
\hline $\mathrm{H}$ & 3.687026000 & 4.460846000 & 0.635822000 \\
\hline $\mathrm{H}$ & 2.443861000 & 1.480676000 & -1.286405000 \\
\hline $\mathrm{H}$ & 3.227092000 & 2.904223000 & -1.991111000 \\
\hline $\mathrm{H}$ & 3.838501000 & 2.204843000 & -0.479532000 \\
\hline $\mathrm{H}$ & 1.029789000 & 4.198837000 & -2.015171000 \\
\hline $\mathrm{H}$ & 0.120115000 & 2.869533000 & -1.266744000 \\
\hline $\mathrm{H}$ & 0.132201000 & 4.462679000 & -0.508000000 \\
\hline $\mathrm{H}$ & -2.148073000 & -0.889245000 & 5.202756000 \\
\hline $\mathrm{H}$ & -3.676673000 & -0.007493000 & 5.125405000 \\
\hline $\mathrm{H}$ & -2.149324000 & 0.876248000 & 5.205045000 \\
\hline $\mathrm{H}$ & -4.748890000 & -0.004780000 & 2.721865000 \\
\hline $\mathrm{H}$ & -5.797714000 & 0.888398000 & 0.685126000 \\
\hline $\mathrm{H}$ & -5.800282000 & -0.886276000 & 0.684255000 \\
\hline $\mathrm{H}$ & -6.057408000 & 0.002091000 & -0.821871000 \\
\hline $\mathrm{H}$ & -2.588279000 & 1.335538000 & -1.209521000 \\
\hline $\mathrm{H}$ & -4.204406000 & 1.273687000 & -1.943739000 \\
\hline $\mathrm{H}$ & -3.940546000 & 2.168798000 & -0.434683000 \\
\hline $\mathrm{H}$ & -3.946383000 & -2.171367000 & -0.434333000 \\
\hline $\mathrm{H}$ & -4.208815000 & -1.275784000 & -1.943295000 \\
\hline $\mathrm{H}$ & -2.592347000 & -1.342027000 & -1.210155000 \\
\hline $\mathrm{H}$ & 1.411758000 & -0.011686000 & -4.902970000 \\
\hline $\mathrm{H}$ & 1.949472000 & -0.906892000 & -3.474073000 \\
\hline $\mathrm{H}$ & -1.828431000 & -1.260673000 & -3.494063000 \\
\hline $\mathrm{H}$ & -0.755238000 & -1.241982000 & -4.908838000 \\
\hline $\mathrm{H}$ & -0.739635000 & 1.247511000 & -4.907772000 \\
\hline $\mathrm{H}$ & -0.267257000 & 2.175216000 & -3.474013000 \\
\hline \multicolumn{4}{|c|}{$\mathrm{Co}^{\mathrm{III}}\left(\mathrm{Tp}^{t \mathrm{Bu}, \mathrm{Me}}\right)\left(\mathrm{N}^{t} \mathrm{Bu}\right), \mathrm{S}=2, \mathrm{C}_{1}, \mathrm{PW} 91$} \\
\hline $\mathrm{Co}$ & 0.075069000 & 0.128981000 & -0.044974000 \\
\hline $\mathrm{N}$ & 0.743967000 & -1.241403000 & 2.473349000 \\
\hline $\mathrm{N}$ & 0.900356000 & -1.478759000 & 1.128027000 \\
\hline $\mathrm{N}$ & 0.743577000 & 1.284140000 & 2.548635000 \\
\hline $\mathrm{N}$ & 0.896441000 & 1.548440000 & 1.214583000 \\
\hline $\mathrm{N}$ & -1.444591000 & 0.022303000 & 2.474188000 \\
\hline $\mathrm{N}$ & -1.728878000 & 0.039577000 & 1.128762000 \\
\hline $\mathrm{N}$ & -0.201948000 & -0.330804000 & -1.735672000 \\
\hline B & 0.003834000 & 0.003977000 & 2.994651000 \\
\hline C & 1.287305000 & -2.302534000 & 4.695994000 \\
\hline $\mathrm{C}$ & 1.287060000 & -2.252135000 & 3.201293000 \\
\hline $\mathrm{C}$ & 1.811036000 & -3.170753000 & 2.302980000 \\
\hline
\end{tabular}




\begin{tabular}{|c|c|c|c|}
\hline C & 1.552409000 & -2.658181000 & 1.018442000 \\
\hline $\mathrm{C}$ & 1.931995000 & -3.293969000 & -0.304162000 \\
\hline C & 0.660825000 & -3.601802000 & -1.118031000 \\
\hline $\mathrm{C}$ & 2.856351000 & -2.340360000 & -1.081909000 \\
\hline C & 2.685793000 & -4.611016000 & -0.054785000 \\
\hline $\mathrm{C}$ & 1.313958000 & 2.281662000 & 4.785573000 \\
\hline $\mathrm{C}$ & 1.308265000 & 2.267307000 & 3.290650000 \\
\hline $\mathrm{C}$ & 1.845125000 & 3.199211000 & 2.404962000 \\
\hline $\mathrm{C}$ & 1.569410000 & 2.718607000 & 1.115459000 \\
\hline C & 1.928501000 & 3.344222000 & -0.216455000 \\
\hline $\mathrm{C}$ & 2.688732000 & 4.660580000 & 0.01035600 \\
\hline $\mathrm{C}$ & 2.829381000 & 2.380455000 & -1.011886000 \\
\hline C & 0.641775000 & 3.643464000 & -1.00829200 \\
\hline C & -2.634338000 & -0.047820000 & 4.697011000 \\
\hline C & -2.591049000 & -0.020235000 & 3.202344000 \\
\hline C & -3.648600000 & -0.030394000 & 2.30445700 \\
\hline C & -3.076134000 & 0.006835000 & 1.01959400 \\
\hline $\mathrm{C}$ & -3.817855000 & 0.010964000 & -0.30243100 \\
\hline C & -5.335095000 & 0.003242000 & -0.051388000 \\
\hline C & -3.457516000 & 1.284610000 & -1.087578000 \\
\hline $\mathrm{C}$ & -3.447659000 & -1.247461000 & -1.11031800 \\
\hline C & -0.109417000 & -0.182238000 & -3.16549800 \\
\hline $\mathrm{C}$ & 1.363981000 & -0.146344000 & -3.63747000 \\
\hline $\mathrm{C}$ & -0.808757000 & -1.381771000 & -3.85431500 \\
\hline $\mathrm{C}$ & -0.797611000 & 1.118062000 & -3.64452900 \\
\hline $\mathrm{H}$ & 1.900904000 & 0.691926000 & -3.178367000 \\
\hline $\mathrm{H}$ & -0.332558000 & -2.325662000 & -3.56416300 \\
\hline $\mathrm{H}$ & -1.862044000 & 1.114187000 & -3.38701500 \\
\hline $\mathrm{H}$ & -0.013310000 & -0.026622000 & 4.19045100 \\
\hline $\mathrm{H}$ & 1.794544000 & -3.215213000 & 5.02614500 \\
\hline $\mathrm{H}$ & 1.811523000 & -1.444320000 & 5.13630700 \\
\hline $\mathrm{H}$ & 0.269282000 & -2.311696000 & 5.10719400 \\
\hline $\mathrm{H}$ & 2.317640000 & -4.094517000 & 2.55594800 \\
\hline $\mathrm{H}$ & 0.932539000 & -4.059175000 & -2.08143200 \\
\hline $\mathrm{H}$ & 0.090609000 & -2.685091000 & -1.30839500 \\
\hline $\mathrm{H}$ & 0.017141000 & -4.306334000 & -0.57321400 \\
\hline $\mathrm{H}$ & 2.363042000 & -1.377556000 & -1.253488000 \\
\hline $\mathrm{H}$ & 3.119477000 & -2.775972000 & -2.05733800 \\
\hline $\mathrm{H}$ & 3.784923000 & -2.158692000 & -0.52328800 \\
\hline $\mathrm{H}$ & 2.062606000 & -5.332521000 & 0.48974800 \\
\hline $\mathrm{H}$ & 2.959752000 & -5.062776000 & -1.01794400 \\
\hline $\mathrm{H}$ & 3.608827000 & -4.447102000 & 0.51710100 \\
\hline $\mathrm{H}$ & 0.296251000 & 2.287607000 & 5.196452000 \\
\hline $\mathrm{H}$ & 1.830226000 & 1.406019000 & 5.19944400 \\
\hline $\mathrm{H}$ & 1.829666000 & 3.180396000 & 5.13961300 \\
\hline $\mathrm{H}$ & 2.368398000 & 4.109138000 & 2.67365700 \\
\hline $\mathrm{H}$ & 2.947263000 & 5.109386000 & -0.95825600 \\
\hline $\mathrm{H}$ & 2.078542000 & 5.384449000 & 0.566240000 \\
\hline $\mathrm{H}$ & 3.621123000 & 4.493791000 & 0.56546000 \\
\hline $\mathrm{H}$ & 2.327795000 & 1.417285000 & -1.17479500 \\
\hline $\mathrm{H}$ & 3.075467000 & 2.810210000 & -1.99372600 \\
\hline $\mathrm{H}$ & 3.765933000 & 2.186054000 & -0.47219500 \\
\hline $\mathrm{H}$ & 0.888646000 & 4.077259000 & -1.98815700 \\
\hline $\mathrm{H}$ & 0.060100000 & 2.727558000 & -1.175164000 \\
\hline $\mathrm{H}$ & 0.004542000 & 4.353381000 & -0.46408900 \\
\hline $\mathrm{H}$ & -2.133377000 & -0.934809000 & 5.10669300 \\
\hline $\mathrm{H}$ & -3.678414000 & -0.065414000 & 5.02729300 \\
\hline
\end{tabular}




$\begin{array}{ll}\mathrm{H} & -2.153042000 \\ \mathrm{H} & -4.701679000 \\ \mathrm{H} & -5.655893000 \\ \mathrm{H} & -5.645993000 \\ \mathrm{H} & -5.864256000 \\ \mathrm{H} & -2.377384000 \\ \mathrm{H} & -3.967893000 \\ \mathrm{H} & -3.765437000 \\ \mathrm{H} & -3.733241000 \\ \mathrm{H} & -3.981254000 \\ \mathrm{H} & -2.369002000 \\ \mathrm{H} & 1.401557000 \\ \mathrm{H} & 1.879836000 \\ \mathrm{H} & -1.867007000 \\ \mathrm{H} & -0.742731000 \\ \mathrm{H} & -0.706165000 \\ \mathrm{H} & -0.330046000\end{array}$

0.834423000

$-0.059290000$

0.885769000

$-0.896130000$

0.010264000

1.339221000

1.288114000

2.182203000

$-2.154902000$

$-1.246853000$

$-1.281370000$

$-0.022471000$

$-1.076786000$

$-1.426802000$

$-1.278165000$

1.207121000

1.998315000
5.138788000

2.557787000

0.517956000

0.496294000

$-1.014070000$

$-1.261115000$

$-2.062252000$

$-0.533630000$

$-0.560320000$

$-2.072852000$

$-1.302499000$

$-4.729842000$

$-3.378175000$

$-3.571698000$

$-4.946946000$

$-4.737107000$

$-3.188129000$

$\begin{array}{lrrr}\mathrm{CO}^{\mathrm{III}}\left(\mathrm{TP}^{t \mathrm{Bu}, \mathrm{Me}}\right)\left(\mathrm{N}^{t} \mathrm{Bu}\right), \mathrm{S}=2, \mathrm{C}_{1}, \mathrm{OLYP} & \\ \mathrm{Co} & 0.081703000 & -0.006369000 & -0.125454000 \\ \mathrm{~N} & 0.745428000 & -1.288937000 & 2.489626000 \\ \mathrm{~N} & 0.919084000 & -1.583260000 & 1.164488000 \\ \mathrm{~N} & 0.742248000 & 1.284484000 & 2.488512000 \\ \mathrm{~N} & 0.914072000 & 1.580399000 & 1.163212000 \\ \mathrm{~N} & -1.463650000 & -0.003468000 & 2.462211000 \\ \mathrm{~N} & -1.788747000 & -0.002850000 & 1.131546000 \\ \mathrm{~N} & -0.161484000 & 0.005334000 & -1.869395000 \\ \mathrm{~B} & 0.003384000 & -0.002703000 & 2.952752000 \\ \mathrm{C} & 1.288471000 & -2.284655000 & 4.761228000 \\ \mathrm{C} & 1.295025000 & -2.262148000 & 3.262796000 \\ \mathrm{C} & 1.841915000 & -3.209029000 & 2.407468000 \\ \mathrm{C} & 1.592429000 & -2.754538000 & 1.102613000 \\ \mathrm{C} & 1.996289000 & -3.428913000 & -0.204614000 \\ \mathrm{C} & 0.734524000 & -3.778004000 & -1.025618000 \\ \mathrm{C} & 2.930178000 & -2.493028000 & -1.004959000 \\ \mathrm{C} & 2.761942000 & -4.737067000 & 0.081985000 \\ \mathrm{C} & 1.278145000 & 2.282945000 & 4.760919000 \\ \mathrm{C} & 1.285952000 & 2.260573000 & 3.262432000 \\ \mathrm{C} & 1.827516000 & 3.210817000 & 2.407787000 \\ \mathrm{C} & 1.580522000 & 2.755681000 & 1.102576000 \\ \mathrm{C} & 1.978465000 & 3.435178000 & -0.203479000 \\ \mathrm{C} & 2.745976000 & 4.742109000 & 0.084625000 \\ \mathrm{C} & 2.908116000 & 2.502604000 & -1.012292000 \\ \mathrm{C} & 0.712224000 & 3.787848000 & -1.016041000 \\ \mathrm{C} & -2.617286000 & -0.002351000 & 4.726377000 \\ \mathrm{C} & -2.588265000 & -0.002306000 & 3.227695000 \\ \mathrm{C} & -3.670484000 & -0.000846000 & 2.363249000 \\ \mathrm{C} & -3.138778000 & -0.001106000 & 1.061390000 \\ \mathrm{C} & -3.930908000 & 0.000579000 & -0.241601000 \\ \mathrm{C} & -5.445683000 & 0.000438000 & 0.052283000 \\ \mathrm{C} & -3.599282000 & 1.275457000 & -1.048385000 \\ \mathrm{C} & -3.599986000 & -1.272399000 & -1.051579000 \\ \mathrm{C} & -0.105444000 & 0.003021000 & -3.305976000 \\ \mathrm{C} & 1.367564000 & -0.007233000 & -3.800694000 \\ \mathrm{C} & -0.818335000 & -1.252563000 & -3.880091000\end{array}$




$\begin{array}{lr}\mathrm{C} & -0.801837000 \\ \mathrm{H} & 1.907018000 \\ \mathrm{H} & -0.347021000 \\ \mathrm{H} & -1.853159000 \\ \mathrm{H} & -0.004594000 \\ \mathrm{H} & 1.805279000 \\ \mathrm{H} & 1.799289000 \\ \mathrm{H} & 0.272851000 \\ \mathrm{H} & 2.353899000 \\ \mathrm{H} & 1.014496000 \\ \mathrm{H} & 0.139933000 \\ \mathrm{H} & 0.099009000 \\ \mathrm{H} & 2.447342000 \\ \mathrm{H} & 3.220295000 \\ \mathrm{H} & 3.843093000 \\ \mathrm{H} & 2.148274000 \\ \mathrm{H} & 3.048005000 \\ \mathrm{H} & 3.678978000 \\ \mathrm{H} & 0.262101000 \\ \mathrm{H} & 1.792250000 \\ \mathrm{H} & 1.790734000 \\ \mathrm{H} & 2.334341000 \\ \mathrm{H} & 3.028883000 \\ \mathrm{H} & 2.134739000 \\ \mathrm{H} & 3.664848000 \\ \mathrm{H} & 2.423907000 \\ \mathrm{H} & 3.192615000 \\ \mathrm{H} & 3.824281000 \\ \mathrm{H} & 0.986757000 \\ \mathrm{H} & 0.116068000 \\ \mathrm{H} & 0.080358000 \\ \mathrm{H} & -2.129646000 \\ \mathrm{H} & -3.657668000 \\ \mathrm{H} & -2.130781000 \\ \mathrm{H} & -4.710710000 \\ \mathrm{H} & -5.751615000 \\ \mathrm{H} & -5.751801000 \\ \mathrm{H} & -6.000914000 \\ \mathrm{H} & -2.534112000 \\ \mathrm{H} & -4.149875000 \\ \mathrm{H} & -3.884027000 \\ \mathrm{H} & -3.884117000 \\ \mathrm{H} & -4.151327000 \\ \mathrm{H} & -2.535072000 \\ \mathrm{H} & 1.395753000 \\ \mathrm{H} & 1.892557000 \\ \mathrm{H} & -1.871560000 \\ \mathrm{H} & -0.765224000 \\ \mathrm{H} & -0.753688000 \\ \mathrm{H} & -0.315006000 \\ \mathrm{H} & \end{array}$

1.268665000
0.873745000

$-2.169566000$

1.303092000

$-0.002799000$

$-3.183757000$

$-1.418421000$

$-2.306301000$

$-4.111178000$

$-4.246272000$

$-2.890773000$

$-4.480896000$

$-1.541779000$

$-2.960222000$

$-2.279616000$

$-5.454035000$

$-5.209187000$

$-4.555557000$

2. 300441000

1. 419047000

3.184322000

4.115694000

5.217026000

5.457774000

4.558209000

1.552188000

2.973066000

2.287597000

4.259776000

2.901517000

4.488920000

$-0.885009000$

$-0.002936000$

0.881030000

0.000331000

0.887391000

$-0.887763000$

0.001629000

1.339234000

1.282823000

2.171297000

$-2.169648000$

$-1.277446000$

$-1.335596000$

$-0.004767000$

$-0.898844000$

$-1.271194000$

$-1.251244000$

1.264013000

2. 178588000
$-3.880259000$

$-3.446224000$

$-3.517831000$

$-3.586059000$

4.141956000

5.106738000

5.193703000

5.169717000

2.710746000

$-1.977324000$

$-1.243296000$

$-0.475324000$

$-1.230052000$

$-1.953980000$

$-0.437780000$

0.636682000

$-0.864393000$

0.651760000

5.168621000

5.194182000

5.106821000

2.711674000

$-0.861286000$

0.643656000

0.650671000

$-1.237494000$

$-1.961363000$

$-0.450952000$

$-1.967518000$

$-1.233080000$

$-0.459217000$

5.153153000

5.061695000

5.152996000

2.655690000

0.616487000

0.614425000

$-0.892313000$

$-1.267108000$

$-1.997327000$

$-0.484742000$

$-0.489878000$

$-2.000076000$

$-1.271574000$

$-4.897281000$

$-3.450654000$

$-3.591206000$

$-4.975829000$

$-4.976149000$

$-3.521503000$

$\mathrm{Cr}^{\text {III }}\left(\right.$ mebp3) $(\mathrm{NMe}), \mathrm{S}=1 / 2, \mathrm{C}_{3 \mathrm{v}}, \mathrm{OLYP}$

$\begin{array}{rrr}\mathrm{H} & -0.513317000 & 0.889091000 \\ \mathrm{H} & 1.026634000 & 0.000000000 \\ \mathrm{H} & -0.513317000 & -0.889091000\end{array}$

4.732596000
4.732596000
4.732596000 


\begin{tabular}{|c|c|c|c|}
\hline C & 0.000000000 & 0.000000000 & 4.331077000 \\
\hline $\mathrm{Cr}$ & 0.000000000 & 0.000000000 & 1.244523000 \\
\hline $\mathrm{P}$ & -1.001209000 & 1.734145000 & -0.150246000 \\
\hline $\mathrm{P}$ & 2.002418000 & 0.000000000 & -0.150246000 \\
\hline $\mathrm{P}$ & -1.001209000 & -1.734145000 & -0.150246000 \\
\hline $\mathrm{C}$ & -0.803135000 & 1.391071000 & -1.963212000 \\
\hline $\mathrm{C}$ & 1.606271000 & 0.000000000 & -1.963212000 \\
\hline $\mathrm{C}$ & -0.803135000 & -1.391071000 & -1.963212000 \\
\hline $\mathrm{H}$ & -1.819465000 & 1.396958000 & -2.389231000 \\
\hline $\mathrm{H}$ & -0.300068000 & 2.274182000 & -2.389231000 \\
\hline $\mathrm{H}$ & -0.300068000 & -2.274182000 & -2.389231000 \\
\hline $\mathrm{H}$ & -1.819465000 & -1.396958000 & -2.389231000 \\
\hline $\mathrm{H}$ & 2.119533000 & -0.877224000 & -2.389231000 \\
\hline $\mathrm{H}$ & 2.119533000 & 0.877224000 & -2.389231000 \\
\hline B & 0.000000000 & 0.000000000 & -2.454164000 \\
\hline C & 3.180063000 & -1.411472000 & 0.125762000 \\
\hline $\mathrm{H}$ & 2.697487000 & -2.362376000 & -0.111868000 \\
\hline $\mathrm{H}$ & 3.491315000 & -1.434764000 & 1.175526000 \\
\hline $\mathrm{H}$ & 4.063638000 & -1.299378000 & -0.513921000 \\
\hline C & 3.180063000 & 1.411472000 & 0.125762000 \\
\hline $\mathrm{H}$ & 2.697487000 & 2.362376000 & -0.111868000 \\
\hline $\mathrm{H}$ & 3.491315000 & 1.434764000 & 1.175526000 \\
\hline $\mathrm{H}$ & 4.063638000 & 1.299378000 & -0.513921000 \\
\hline $\mathrm{C}$ & -0.367661000 & 3.459751000 & 0.125762000 \\
\hline $\mathrm{H}$ & 0.697134000 & 3.517280000 & -0.111868000 \\
\hline $\mathrm{H}$ & -0.503116000 & 3.740949000 & 1.175526000 \\
\hline $\mathrm{H}$ & -0.906524000 & 4.168903000 & -0.513921000 \\
\hline C & -2.812402000 & 2.048280000 & 0.125762000 \\
\hline $\mathrm{H}$ & -3.394621000 & 1.154904000 & -0.111868000 \\
\hline $\mathrm{H}$ & -2.988199000 & 2.306186000 & 1.175526000 \\
\hline $\mathrm{H}$ & -3.157113000 & 2.869524000 & -0.513921000 \\
\hline $\mathrm{C}$ & -2.812402000 & -2.048280000 & 0.125762000 \\
\hline $\mathrm{H}$ & -3.394621000 & -1.154904000 & -0.111868000 \\
\hline $\mathrm{H}$ & -2.988199000 & -2.306186000 & 1.175526000 \\
\hline $\mathrm{H}$ & -3.157113000 & -2.869524000 & -0.513921000 \\
\hline C & -0.367661000 & -3.459751000 & 0.125762000 \\
\hline $\mathrm{H}$ & 0.697134000 & -3.517280000 & -0.111868000 \\
\hline $\mathrm{H}$ & -0.503116000 & -3.740949000 & 1.175526000 \\
\hline $\mathrm{H}$ & -0.906524000 & -4.168903000 & -0.513921000 \\
\hline $\mathrm{N}$ & 0.000000000 & 0.000000000 & 2.902935000 \\
\hline $\mathrm{C}$ & 0.000000000 & 0.000000000 & -4.120968000 \\
\hline $\mathrm{H}$ & -0.506831000 & 0.877856000 & -4.550241000 \\
\hline $\mathrm{H}$ & -0.506831000 & -0.877856000 & -4.550241000 \\
\hline $\mathrm{H}$ & 1.013661000 & 0.000000000 & -4.550241000 \\
\hline \multicolumn{4}{|c|}{$\operatorname{Cr}^{\text {III }}\left(\right.$ mebp3) $(\mathrm{NMe}), \mathrm{S}=3 / 2, \mathrm{C}_{\mathrm{S}}, \mathrm{OLYP}$} \\
\hline $\mathrm{H}$ & -4.823525000 & 0.585083000 & 0.888154000 \\
\hline $\mathrm{H}$ & -4.844255000 & -0.953076000 & 0.000000000 \\
\hline $\mathrm{H}$ & -4.823525000 & 0.585083000 & -0.888154000 \\
\hline C & -4.423680000 & 0.066897000 & 0.000000000 \\
\hline Cr & -1.309824000 & 0.021237000 & 0.000000000 \\
\hline $\mathrm{P}$ & 0.196836000 & 1.001603000 & 1.738610000 \\
\hline $\mathrm{P}$ & 0.145737000 & -2.012083000 & 0.000000000 \\
\hline P & 0.196836000 & 1.001603000 & -1.738610000 \\
\hline C & 2.002539000 & 0.771909000 & 1.390775000 \\
\hline
\end{tabular}




$\begin{array}{lrrr}\mathrm{C} & 1.961579000 & -1.638315000 & 0.000000000 \\ \mathrm{C} & 2.002539000 & 0.771909000 & -1.390775000 \\ \mathrm{H} & 2.446792000 & 1.780224000 & 1.394920000 \\ \mathrm{H} & 2.422887000 & 0.263335000 & 2.273204000 \\ \mathrm{H} & 2.422887000 & 0.263335000 & -2.273204000 \\ \mathrm{H} & 2.446792000 & 1.780224000 & -1.394920000 \\ \mathrm{H} & 2.381510000 & -2.157613000 & -0.876392000 \\ \mathrm{H} & 2.381510000 & -2.157613000 & 0.876392000 \\ \mathrm{~B} & 2.479068000 & -0.040150000 & 0.00000000 \\ \mathrm{C} & -0.144253000 & -3.186043000 & -1.411145000 \\ \mathrm{H} & 0.098134000 & -2.705468000 & -2.362019000 \\ \mathrm{H} & -1.197681000 & -3.485147000 & -1.435165000 \\ \mathrm{H} & 0.484385000 & -4.077745000 & -1.301277000 \\ \mathrm{C} & -0.144253000 & -3.186043000 & 1.411145000 \\ \mathrm{H} & 0.098134000 & -2.705468000 & 2.362019000 \\ \mathrm{H} & -1.197681000 & -3.485147000 & 1.435165000 \\ \mathrm{H} & 0.484385000 & -4.077745000 & 1.301277000 \\ \mathrm{C} & -0.085643000 & 0.374341000 & 3.465166000 \\ \mathrm{H} & 0.134382000 & -0.694344000 & 3.522887000 \\ \mathrm{H} & -1.132644000 & 0.526250000 & 3.748837000 \\ \mathrm{H} & 0.563176000 & 0.902368000 & 4.174111000 \\ \mathrm{C} & -0.047570000 & 2.817030000 & 2.053682000 \\ \mathrm{H} & 0.195468000 & 3.395285000 & 1.159040000 \\ \mathrm{H} & -1.093451000 & 3.010868000 & 2.315140000 \\ \mathrm{H} & 0.599896000 & 3.153220000 & 2.872294000 \\ \mathrm{C} & -0.047570000 & 2.817030000 & -2.053682000 \\ \mathrm{H} & 0.195468000 & 3.395285000 & -1.159040000 \\ \mathrm{H} & -1.093451000 & 3.010868000 & -2.315140000 \\ \mathrm{H} & 0.599896000 & 3.153220000 & -2.872294000 \\ \mathrm{C} & -0.085643000 & 0.374341000 & -3.465166000 \\ \mathrm{H} & 0.134382000 & -0.694344000 & -3.522887000 \\ \mathrm{H} & -1.132644000 & 0.526250000 & -3.748837000 \\ \mathrm{H} & 0.563176000 & 0.902368000 & -4.174111000 \\ \mathrm{~N} & -2.999665000 & 0.047542000 & 0.000000000 \\ \mathrm{C} & 4.145404000 & -0.067648000 & 0.000000000 \\ \mathrm{H} & 4.583064000 & 0.432203000 & 0.877792000 \\ \mathrm{H} & 4.583064000 & 0.432203000 & -0.877792000 \\ \mathrm{H} & 4.558440000 & -1.088074000 & 0.000000000 \\ & & & \end{array}$

$\mathrm{Mn}^{\text {III }}$ (mebp3) (NMe), $\mathrm{S}=0, \mathrm{C}_{S}, \mathrm{OLYP}$

$\begin{array}{lrrr}\mathrm{H} & -0.513469000 & 0.889355000 & 4.522360000 \\ \mathrm{H} & 1.026939000 & 0.000000000 & 4.522360000 \\ \mathrm{H} & -0.513469000 & -0.889355000 & 4.522360000 \\ \mathrm{C} & 0.000000000 & 0.000000000 & 4.121277000 \\ \mathrm{Mn} & 0.000000000 & 0.000000000 & 1.060730000 \\ \mathrm{P} & -0.980187000 & 1.697733000 & -0.100472000 \\ \mathrm{P} & 1.960373000 & 0.000000000 & -0.100472000 \\ \mathrm{P} & -0.980187000 & -1.697733000 & -0.100472000 \\ \mathrm{C} & -0.801398000 & 1.388063000 & -1.920162000 \\ \mathrm{C} & 1.602797000 & 0.000000000 & -1.920162000 \\ \mathrm{C} & -0.801398000 & -1.388063000 & -1.920162000 \\ \mathrm{H} & -1.822496000 & 1.398335000 & -2.334298000 \\ \mathrm{H} & -0.299746000 & 2.277495000 & -2.334298000 \\ \mathrm{H} & -0.299746000 & -2.277495000 & -2.334298000 \\ \mathrm{H} & -1.822496000 & -1.398335000 & -2.334298000\end{array}$




\begin{tabular}{|c|c|}
\hline $\mathrm{H}$ & 2.122242000 \\
\hline $\mathrm{H}$ & 2.122242000 \\
\hline B & 0.000000000 \\
\hline $\mathrm{C}$ & 3.140090000 \\
\hline $\mathrm{H}$ & 2.678241000 \\
\hline $\mathrm{H}$ & 3.405072000 \\
\hline $\mathrm{H}$ & 4.051634000 \\
\hline $\mathrm{C}$ & 3.140090000 \\
\hline $\mathrm{H}$ & 2.678241000 \\
\hline $\mathrm{H}$ & 3.405072000 \\
\hline $\mathrm{H}$ & 4.051634000 \\
\hline C & -0.352696000 \\
\hline $\mathrm{H}$ & 0.704215000 \\
\hline $\mathrm{H}$ & -0.462849000 \\
\hline $\mathrm{H}$ & -0.917204000 \\
\hline C & -2.787394000 \\
\hline $\mathrm{H}$ & -3.382455000 \\
\hline $\mathrm{H}$ & -2.942223000 \\
\hline $\mathrm{H}$ & -3.134430000 \\
\hline $\mathrm{C}$ & -2.787394000 \\
\hline $\mathrm{H}$ & -3.382455000 \\
\hline $\mathrm{H}$ & -2.942223000 \\
\hline $\mathrm{H}$ & -3.134430000 \\
\hline C & -0.352696000 \\
\hline $\mathrm{H}$ & 0.704215000 \\
\hline $\mathrm{H}$ & -0.462849000 \\
\hline $\mathrm{H}$ & -0.917204000 \\
\hline $\mathrm{N}$ & 0.000000000 \\
\hline C & 0.000000000 \\
\hline $\mathrm{H}$ & -0.506824000 \\
\hline $\mathrm{H}$ & -0.506824000 \\
\hline $\mathrm{H}$ & 1.013649000 \\
\hline
\end{tabular}

$$
\begin{array}{r}
-0.879160000 \\
0.879160000 \\
0.000000000 \\
-1.405674000 \\
-2.359440000 \\
-1.431467000 \\
-1.280116000 \\
1.405674000 \\
2.359440000 \\
1.431467000 \\
1.280116000 \\
3.422235000 \\
3.499144000 \\
3.664613000 \\
4.148876000 \\
2.016561000 \\
1.139704000 \\
2.233145000 \\
2.868760000 \\
-2.016561000 \\
-1.139704000 \\
-2.233145000 \\
-2.868760000 \\
-3.422235000 \\
-3.499144000 \\
-3.664613000 \\
-4.148876000 \\
0.000000000 \\
0.000000000 \\
0.877846000 \\
-0.877846000 \\
0.000000000
\end{array}
$$

$$
\begin{array}{r}
-2.334298000 \\
-2.334298000 \\
-2.422776000 \\
0.216047000 \\
-0.047620000 \\
1.278553000 \\
-0.380542000 \\
0.216047000 \\
-0.047620000 \\
1.278553000 \\
-0.380542000 \\
0.216047000 \\
-0.047620000 \\
1.278553000 \\
-0.380542000 \\
0.216047000 \\
-0.047620000 \\
1.278553000 \\
-0.380542000 \\
0.216047000 \\
-0.047620000 \\
1.278553000 \\
-0.380542000 \\
0.216047000 \\
-0.047620000 \\
1.278553000 \\
-0.380542000 \\
2.697391000 \\
-4.088324000 \\
-4.517295000 \\
-4.517295000 \\
-4.517295000
\end{array}
$$

$\mathrm{Mn}^{\text {III }}$ (mebp3) (NMe), $\mathrm{S}=1, \mathrm{C}_{\mathrm{S}}$, OLYP

$\begin{array}{lrrr}\mathrm{H} & -4.639787000 & -0.500282000 & 0.887737000 \\ \mathrm{H} & -4.006683000 & -1.903413000 & 0.000000000 \\ \mathrm{H} & -4.639787000 & -0.500282000 & -0.887737000 \\ \mathrm{C} & -4.058901000 & -0.801471000 & 0.000000000 \\ \mathrm{Mn} & -1.144045000 & 0.144669000 & 0.000000000 \\ \mathrm{P} & 0.213272000 & 1.028600000 & 1.727502000 \\ \mathrm{P} & -0.054686000 & -1.844619000 & 0.000000000 \\ \mathrm{P} & 0.213272000 & 1.028600000 & -1.727502000 \\ \mathrm{C} & 2.012177000 & 0.764847000 & 1.391628000 \\ \mathrm{C} & 1.786569000 & -1.622551000 & 0.000000000 \\ \mathrm{C} & 2.012177000 & 0.764847000 & -1.391628000 \\ \mathrm{H} & 2.484647000 & 1.760243000 & 1.391870000 \\ \mathrm{H} & 2.418762000 & 0.242819000 & 2.272623000 \\ \mathrm{H} & 2.418762000 & 0.242819000 & -2.272623000 \\ \mathrm{H} & 2.484647000 & 1.760243000 & -1.391870000 \\ \mathrm{H} & 2.158117000 & -2.175783000 & -0.877365000 \\ \mathrm{H} & 2.158117000 & -2.175783000 & 0.877365000 \\ \mathrm{~B} & 2.437263000 & -0.075768000 & 0.000000000 \\ \mathrm{C} & -0.436014000 & -3.018500000 & -1.397826000 \\ \mathrm{H} & -0.156177000 & -2.578774000 & -2.357349000 \\ \mathrm{H} & -1.510254000 & -3.231442000 & -1.418342000\end{array}$




$\begin{array}{lr}\mathrm{H} & 0.113890000 \\ \mathrm{C} & -0.436014000 \\ \mathrm{H} & -0.156177000 \\ \mathrm{H} & -1.510254000 \\ \mathrm{H} & 0.113890000 \\ \mathrm{C} & -0.123998000 \\ \mathrm{H} & 0.113976000 \\ \mathrm{H} & -1.182234000 \\ \mathrm{H} & 0.490838000 \\ \mathrm{C} & -0.001620000 \\ \mathrm{H} & 0.252443000 \\ \mathrm{H} & -1.045045000 \\ \mathrm{H} & 0.647840000 \\ \mathrm{C} & -0.001620000 \\ \mathrm{H} & 0.252443000 \\ \mathrm{H} & -1.045045000 \\ \mathrm{H} & 0.647840000 \\ \mathrm{C} & -0.123998000 \\ \mathrm{H} & 0.113976000 \\ \mathrm{H} & -1.182234000 \\ \mathrm{H} & 0.490838000 \\ \mathrm{~N} & -2.768870000 \\ \mathrm{C} & 4.095683000 \\ \mathrm{H} & 4.568903000 \\ \mathrm{H} & 4.568903000 \\ \mathrm{H} & 4.431629000 \\ & \end{array}$

$$
\begin{array}{r}
-3.958706000 \\
-3.018500000 \\
-2.578774000 \\
-3.231442000 \\
-3.958706000 \\
0.387775000 \\
-0.675853000 \\
0.522912000 \\
0.926723000 \\
2.847776000 \\
3.433692000 \\
3.054697000 \\
3.164640000 \\
2.847776000 \\
3.433692000 \\
3.054697000 \\
3.164640000 \\
0.387775000 \\
-0.675853000 \\
0.522912000 \\
0.926723000 \\
-0.199803000 \\
-0.225396000 \\
0.240811000 \\
0.240811000 \\
-1.273682000
\end{array}
$$
$-1.270198000$
1. 397826000
2.357349000
1.418342000
1. 270198000
3.437659000
3.500642000
3.684874000
4.168072000
2.064130000
1.177342000
2. 326572000
2.888989000
$-2.064130000$
$-1.177342000$
$-2.326572000$
$-2.888989000$
$-3.437659000$
$-3.500642000$
$-3.684874000$
$-4.168072000$
0.000000000
0.000000000
0.877753000
$-0.877753000$
0.000000000

$\mathrm{Mn}^{\text {III }}$ (mebp3) (NMe), $\mathrm{S}=2, \mathrm{C}_{\mathrm{S}}, \mathrm{OLYP}$

$\begin{array}{lr}\mathrm{H} & -4.853721000 \\ \mathrm{H} & -4.885478000 \\ \mathrm{H} & -4.853721000 \\ \mathrm{C} & -4.438587000 \\ \mathrm{Mn} & -1.290312000 \\ \mathrm{P} & 0.189551000 \\ \mathrm{P} & 0.149368000 \\ \mathrm{P} & 0.189551000 \\ \mathrm{C} & 1.984495000 \\ \mathrm{C} & 1.943703000 \\ \mathrm{C} & 1.984495000 \\ \mathrm{H} & 2.443206000 \\ \mathrm{H} & 2.421635000 \\ \mathrm{H} & 2.421635000 \\ \mathrm{H} & 2.443206000 \\ \mathrm{H} & 2.377789000 \\ \mathrm{H} & 2.377789000 \\ \mathrm{~B} & 2.439679000 \\ \mathrm{C} & -0.135749000 \\ \mathrm{H} & 0.099279000 \\ \mathrm{H} & -1.187708000 \\ \mathrm{H} & 0.498269000 \\ \mathrm{C} & -0.135749000 \\ \mathrm{H} & 0.099279000 \\ \mathrm{H} & -1.187708000 \\ \mathrm{H} & 0.498269000 \\ \mathrm{C} & -0.046154000\end{array}$

$$
\begin{array}{r}
0.676105000 \\
-0.844710000 \\
0.676105000 \\
0.167797000 \\
-0.073461000 \\
1.048042000 \\
-2.081065000 \\
1.048042000 \\
0.791098000 \\
-1.632210000 \\
0.791098000 \\
1.792583000 \\
0.274673000 \\
0.274673000 \\
1.792583000 \\
-2.139252000 \\
-2.139252000 \\
-0.018770000 \\
-3.236429000 \\
-2.736295000 \\
-3.540363000 \\
-4.125893000 \\
-3.236429000 \\
-2.736295000 \\
-3.540363000 \\
-4.125893000 \\
0.431298000
\end{array}
$$

0.895039000

0.000000000

$-0.895039000$

0.000000000

0.000000000

1. 807836000

0.000000000

$-1.807836000$

1.402719000

0.000000000

$-1.402719000$

1. 403063000

2. 272227000

$-2.272227000$

$-1.403063000$

$-0.876378000$

0.876378000

0.000000000

$-1.421738000$

$-2.364823000$

$-1.446580000$

$-1.328346000$

1. 421738000

2. 364823000

1. 446580000

1. 328346000

3. 544910000 


$\begin{array}{lr}\mathrm{H} & 0.169668000 \\ \mathrm{H} & -1.086119000 \\ \mathrm{H} & 0.619904000 \\ \mathrm{C} & -0.020991000 \\ \mathrm{H} & 0.213192000 \\ \mathrm{H} & -1.060089000 \\ \mathrm{H} & 0.643431000 \\ \mathrm{C} & -0.020991000 \\ \mathrm{H} & 0.213192000 \\ \mathrm{H} & -1.060089000 \\ \mathrm{H} & 0.643431000 \\ \mathrm{C} & -0.046154000 \\ \mathrm{H} & 0.169668000 \\ \mathrm{H} & -1.086119000 \\ \mathrm{H} & 0.619904000 \\ \mathrm{~N} & -3.034879000 \\ \mathrm{C} & 4.106878000 \\ \mathrm{H} & 4.545995000 \\ \mathrm{H} & 4.545995000 \\ \mathrm{H} & 4.515414000 \\ & \end{array}$

$$
\begin{array}{r}
-0.639263000 \\
0.585611000 \\
0.955769000 \\
2.869944000 \\
3.431681000 \\
3.081684000 \\
3.211800000 \\
2.869944000 \\
3.431681000 \\
3.081684000 \\
3.211800000 \\
0.431298000 \\
-0.639263000 \\
0.585611000 \\
0.955769000 \\
0.056047000 \\
-0.056097000 \\
0.441432000 \\
0.441432000 \\
-1.078261000
\end{array}
$$
3.598084000
3.853125000
4.240643000
2.107486000
1.199479000
2.382446000
2. 910501000
$-2.107486000$
$-1.199479000$
$-2.382446000$
$-2.910501000$
$-3.544910000$
$-3.598084000$
$-3.853125000$
$-4.240643000$
0.000000000
0.000000000
0.877969000
$-0.877969000$
0.000000000

$\mathrm{Fe}^{\text {III }}($ mebp 3$)(\mathrm{NMe}), \mathrm{S}=1 / 2, \mathrm{C}_{\mathrm{S}}, \mathrm{OLYP}$

$\begin{array}{lrrr}\mathrm{H} & -4.406945000 & 0.725258000 & 0.891257000 \\ \mathrm{H} & -4.657062000 & -0.789882000 & 0.000000000 \\ \mathrm{H} & -4.406945000 & 0.725258000 & -0.891257000 \\ \mathrm{C} & -4.090134000 & 0.157079000 & 0.000000000 \\ \mathrm{Fe} & -1.048052000 & 0.020752000 & 0.000000000 \\ \mathrm{P} & 0.126141000 & 0.945754000 & 1.642521000 \\ \mathrm{P} & 0.087790000 & -1.909764000 & 0.000000000 \\ \mathrm{P} & 0.126141000 & 0.945754000 & -1.642521000 \\ \mathrm{C} & 1.948422000 & 0.768449000 & 1.386279000 \\ \mathrm{C} & 1.910850000 & -1.623448000 & 0.000000000 \\ \mathrm{C} & 1.948422000 & 0.768449000 & -1.386279000 \\ \mathrm{H} & 2.368989000 & 1.786783000 & 1.404947000 \\ \mathrm{H} & 2.347418000 & 0.262028000 & 2.279841000 \\ \mathrm{H} & 2.347418000 & 0.262028000 & -2.279841000 \\ \mathrm{H} & 2.368989000 & 1.786783000 & -1.404947000 \\ \mathrm{H} & 2.307994000 & -2.153855000 & -0.880058000 \\ \mathrm{H} & 2.307994000 & -2.153855000 & 0.880058000 \\ \mathrm{~B} & 2.452896000 & -0.032943000 & 0.000000000 \\ \mathrm{C} & -0.293565000 & -3.068662000 & -1.403457000 \\ \mathrm{H} & 0.000116000 & -2.629513000 & -2.358742000 \\ \mathrm{H} & -1.370078000 & -3.267804000 & -1.429722000 \\ \mathrm{H} & 0.247497000 & -4.013278000 & -1.271198000 \\ \mathrm{C} & -0.293565000 & -3.068662000 & 1.403457000 \\ \mathrm{H} & 0.000116000 & -2.629513000 & 2.358742000 \\ \mathrm{H} & -1.370078000 & -3.267804000 & 1.429722000 \\ \mathrm{H} & 0.247497000 & -4.013278000 & 1.271198000 \\ \mathrm{C} & -0.234507000 & 0.336811000 & 3.366044000 \\ \mathrm{H} & 0.031408000 & -0.717256000 & 3.467076000 \\ \mathrm{H} & -1.303683000 & 0.444777000 & 3.578093000 \\ \mathrm{H} & 0.340317000 & 0.912612000 & 4.101298000 \\ \mathrm{C} & -0.202727000 & 2.754132000 & 1.948005000 \\ \mathrm{H} & 0.104568000 & 3.356265000 & 1.091136000 \\ \mathrm{H} & -1.273199000 & 2.912475000 & 2.118487000 \\ & & & \end{array}$




$\begin{array}{rr}\mathrm{H} & 0.354392000 \\ \mathrm{C} & -0.202727000 \\ \mathrm{H} & 0.104568000 \\ \mathrm{H} & -1.273199000 \\ \mathrm{H} & 0.354392000 \\ \mathrm{C} & -0.234507000 \\ \mathrm{H} & 0.031408000 \\ \mathrm{H} & -1.303683000 \\ \mathrm{H} & 0.340317000 \\ \mathrm{~N} & -2.690937000 \\ \mathrm{C} & 4.115966000 \\ \mathrm{H} & 4.552805000 \\ \mathrm{H} & 4.552805000 \\ \mathrm{H} & 4.528520000\end{array}$

$$
\begin{array}{r}
3.093152000 \\
2.754132000 \\
3.356265000 \\
2.912475000 \\
3.093152000 \\
0.336811000 \\
-0.717256000 \\
0.444777000 \\
0.912612000 \\
-0.096838000 \\
-0.061530000 \\
0.438252000 \\
0.438252000 \\
-1.082114000
\end{array}
$$

\begin{tabular}{|c|c|c|c|}
\hline $\mathrm{H}$ & -4.686588000 & -0.666490000 & 0.894180000 \\
\hline $\mathrm{H}$ & -3.966815000 & -2.009144000 & 0.000000000 \\
\hline $\mathrm{H}$ & -4.686588000 & -0.666490000 & -0.894180000 \\
\hline $\mathrm{C}$ & -4.078114000 & -0.909414000 & 0.000000000 \\
\hline $\mathrm{Fe}$ & -1.159019000 & 0.104829000 & 0.000000000 \\
\hline $\mathrm{P}$ & 0.225027000 & 1.066299000 & 1.811829000 \\
\hline $\mathrm{P}$ & -0.089946000 & -1.879207000 & 0.000000000 \\
\hline $\mathrm{P}$ & 0.225027000 & 1.066299000 & -1.811829000 \\
\hline $\mathrm{C}$ & 2.011043000 & 0.779175000 & 1.400641000 \\
\hline $\mathrm{C}$ & 1.741613000 & -1.612760000 & 0.000000000 \\
\hline $\mathrm{C}$ & 2.011043000 & 0.779175000 & -1.400641000 \\
\hline $\mathrm{H}$ & 2.496929000 & 1.767920000 & 1.390036000 \\
\hline $\mathrm{H}$ & 2.442140000 & 0.252884000 & 2.267187000 \\
\hline $\mathrm{H}$ & 2.442140000 & 0.252884000 & -2.267187000 \\
\hline $\mathrm{H}$ & 2.496929000 & 1.767920000 & -1.390036000 \\
\hline $\mathrm{H}$ & 2.118042000 & -2.161270000 & -0.878127000 \\
\hline $\mathrm{H}$ & 2.118042000 & -2.161270000 & 0.878127000 \\
\hline B & 2.404106000 & -0.062207000 & 0.000000000 \\
\hline $\mathrm{C}$ & -0.477130000 & -3.021821000 & -1.410739000 \\
\hline $\mathrm{H}$ & -0.214063000 & -2.553382000 & -2.361650000 \\
\hline $\mathrm{H}$ & -1.549230000 & -3.243804000 & -1.421799000 \\
\hline $\mathrm{H}$ & 0.085595000 & -3.957642000 & -1.309603000 \\
\hline $\mathrm{C}$ & -0.477130000 & -3.021821000 & 1.410739000 \\
\hline $\mathrm{H}$ & -0.214063000 & -2.553382000 & 2.361650000 \\
\hline $\mathrm{H}$ & -1.549230000 & -3.243804000 & 1.421799000 \\
\hline $\mathrm{H}$ & 0.085595000 & -3.957642000 & 1.309603000 \\
\hline $\mathrm{C}$ & -0.014700000 & 0.457834000 & 3.550926000 \\
\hline $\mathrm{H}$ & 0.196137000 & -0.612718000 & 3.610761000 \\
\hline $\mathrm{H}$ & -1.051649000 & 0.622559000 & 3.863064000 \\
\hline $\mathrm{H}$ & 0.658831000 & 0.984001000 & 4.238385000 \\
\hline $\mathrm{C}$ & 0.047334000 & 2.892465000 & 2.119869000 \\
\hline $\mathrm{H}$ & 0.251005000 & 3.454153000 & 1.204398000 \\
\hline $\mathrm{H}$ & -0.977472000 & 3.115408000 & 2.437292000 \\
\hline $\mathrm{H}$ & 0.746185000 & 3.225872000 & 2.896856000 \\
\hline $\mathrm{C}$ & 0.047334000 & 2.892465000 & -2.119869000 \\
\hline $\mathrm{H}$ & 0.251005000 & 3.454153000 & -1.204398000 \\
\hline $\mathrm{H}$ & -0.977472000 & 3.115408000 & -2.437292000 \\
\hline $\mathrm{H}$ & 0.746185000 & 3.225872000 & -2.896856000 \\
\hline $\mathrm{C}$ & -0.014700000 & 0.457834000 & -3.550926000 \\
\hline
\end{tabular}

2.829273000
-1.948005000
-1.091136000
-2.118487000
-2.829273000
-3.366044000
-3.467076000
-3.578093000
-4.101298000
0.000000000
0.000000000
0.878058000
-0.878058000
0.000000000

$F e^{I I I}\left(\right.$ mebp3) (NMe), $S=3 / 2, C_{S}$, OLYP 


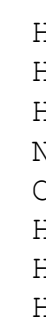

$\mathrm{Fe}^{\text {III }}$ (mebp3) (NMe), $\mathrm{S}=5 / 2, \mathrm{C}_{\mathrm{S}}, \mathrm{OLYP}$

$$
\begin{array}{r}
-0.612718000 \\
0.622559000 \\
0.984001000 \\
-0.280543000 \\
-0.249953000 \\
0.205887000 \\
0.205887000 \\
-1.304963000
\end{array}
$$

-3.610761000
-3.863064000
-4.238385000
0.000000000
0.000000000
0.877753000
-0.877753000
0.000000000

$\begin{array}{lrrr}\mathrm{H} & -4.847792000 & 0.606573000 & 0.886545000 \\ \mathrm{H} & -4.886440000 & -0.928110000 & 0.000000000 \\ \mathrm{H} & -4.847792000 & 0.606573000 & -0.886545000 \\ \mathrm{C} & -4.432347000 & 0.084216000 & 0.00000000 \\ \mathrm{Fe} & -1.253065000 & 0.019460000 & 0.00000000 \\ \mathrm{P} & 0.171794000 & 1.055561000 & 1.834640000 \\ \mathrm{P} & 0.122990000 & -2.124460000 & 0.000000000 \\ \mathrm{P} & 0.171794000 & 1.055561000 & -1.834640000 \\ \mathrm{C} & 1.957480000 & 0.778132000 & 1.401966000 \\ \mathrm{C} & 1.920283000 & -1.650136000 & 0.000000000 \\ \mathrm{C} & 1.957480000 & 0.778132000 & -1.401966000 \\ \mathrm{H} & 2.422326000 & 1.776952000 & 1.394894000 \\ \mathrm{H} & 2.398888000 & 0.262178000 & 2.269564000 \\ \mathrm{H} & 2.398888000 & 0.262178000 & -2.269564000 \\ \mathrm{H} & 2.422326000 & 1.776952000 & -1.394894000 \\ \mathrm{H} & 2.361931000 & -2.153752000 & -0.874649000 \\ \mathrm{H} & 2.361931000 & -2.153752000 & 0.874649000 \\ \mathrm{~B} & 2.408721000 & -0.038464000 & 0.000000000 \\ \mathrm{C} & -0.100665000 & -3.299632000 & -1.419005000 \\ \mathrm{H} & 0.093391000 & -2.788251000 & -2.365700000 \\ \mathrm{H} & -1.130666000 & -3.671947000 & -1.435006000 \\ \mathrm{H} & 0.590959000 & -4.146311000 & -1.331761000 \\ \mathrm{C} & -0.100665000 & -3.299632000 & 1.419005000 \\ \mathrm{H} & 0.093391000 & -2.788251000 & 2.365700000 \\ \mathrm{H} & -1.130666000 & -3.671947000 & 1.435006000 \\ \mathrm{H} & 0.590959000 & -4.146311000 & 1.331761000 \\ \mathrm{C} & -0.045002000 & 0.423332000 & 3.566360000 \\ \mathrm{H} & 0.122881000 & -0.656656000 & 3.597773000 \\ \mathrm{H} & -1.066140000 & 0.621738000 & 3.909292000 \\ \mathrm{H} & 0.667176000 & 0.906933000 & 4.245912000 \\ \mathrm{C} & -0.008456000 & 2.877149000 & 2.143220000 \\ \mathrm{H} & 0.183575000 & 3.436343000 & 1.223523000 \\ \mathrm{H} & -1.029203000 & 3.098765000 & 2.472504000 \\ \mathrm{H} & 0.701554000 & 3.211331000 & 2.909329000 \\ \mathrm{C} & -0.008456000 & 2.877149000 & -2.143220000 \\ \mathrm{H} & 0.183575000 & 3.436343000 & -1.223523000 \\ \mathrm{H} & -1.029203000 & 3.098765000 & -2.472504000 \\ \mathrm{H} & 0.701554000 & 3.211331000 & -2.909329000 \\ \mathrm{C} & -0.045002000 & 0.423332000 & -3.566360000 \\ \mathrm{H} & 0.122881000 & -0.656656000 & -3.597773000 \\ \mathrm{H} & -1.066140000 & 0.621738000 & -3.909292000 \\ \mathrm{H} & 0.667176000 & 0.906933000 & -4.245912000 \\ \mathrm{~N} & -3.026584000 & 0.048607000 & 0.000000000 \\ \mathrm{C} & 4.078174000 & -0.063123000 & 0.000000000 \\ \mathrm{H} & 4.515270000 & 0.437324000 & 0.877483000\end{array}$




\begin{tabular}{|c|c|c|c|}
\hline $\mathrm{H}$ & 4.515270000 & 0.437324000 & -0.877483000 \\
\hline $\mathrm{H}$ & 4.493249000 & -1.082504000 & 0.000000000 \\
\hline $\mathrm{Cr}^{\mathrm{I}}$ &,$\left.{ }^{\mathrm{Me}}\right)\left(\mathrm{N}^{t} \mathrm{Bu}\right), \mathrm{S}$ & $1 / 2, \quad C_{S}, \quad O L Y P$ & \\
\hline $\mathrm{Cr}$ & -0.704854000 & 0.002149000 & 0.000000000 \\
\hline $\mathrm{N}$ & 1.938801000 & 0.744941000 & -1.271492000 \\
\hline $\mathrm{N}$ & 0.604370000 & 0.894340000 & -1.537290000 \\
\hline $\mathrm{N}$ & 1.938801000 & 0.744941000 & 1.271492000 \\
\hline $\mathrm{N}$ & 0.604370000 & 0.894340000 & 1.537290000 \\
\hline $\mathrm{N}$ & 1.942741000 & -1.460028000 & 0.000000000 \\
\hline $\mathrm{N}$ & 0.610211000 & -1.774795000 & 0.000000000 \\
\hline $\mathrm{N}$ & -2.378693000 & -0.027442000 & 0.000000000 \\
\hline B & 2.424395000 & 0.011646000 & 0.000000000 \\
\hline $\mathrm{C}$ & 4.185606000 & 1.336451000 & -2.287439000 \\
\hline $\mathrm{C}$ & 2.688175000 & 1.311284000 & -2.252269000 \\
\hline $\mathrm{C}$ & 1.808556000 & 1.842916000 & -3.182534000 \\
\hline $\mathrm{C}$ & 0.513802000 & 1.568108000 & -2.712604000 \\
\hline $\mathrm{C}$ & -0.794728000 & 1.956855000 & -3.395292000 \\
\hline $\mathrm{C}$ & -1.600008000 & 0.686383000 & -3.748566000 \\
\hline $\mathrm{C}$ & -1.608858000 & 2.893357000 & -2.474951000 \\
\hline $\mathrm{C}$ & -0.506998000 & 2.715899000 & -4.708302000 \\
\hline C & 4.185606000 & 1.336451000 & 2.287439000 \\
\hline C & 2.688175000 & 1.311284000 & 2.252269000 \\
\hline $\mathrm{C}$ & 1.808556000 & 1.842916000 & 3.182534000 \\
\hline $\mathrm{C}$ & 0.513802000 & 1.568108000 & 2.712604000 \\
\hline $\mathrm{C}$ & -0.794728000 & 1.956855000 & 3.395292000 \\
\hline $\mathrm{C}$ & -0.506998000 & 2.715899000 & 4.708302000 \\
\hline $\mathrm{C}$ & -1.608858000 & 2.893357000 & 2.474951000 \\
\hline C & -1.600008000 & 0.686383000 & 3.748566000 \\
\hline $\mathrm{C}$ & 4.196476000 & -2.622192000 & 0.000000000 \\
\hline $\mathrm{C}$ & 2.698633000 & -2.587382000 & 0.000000000 \\
\hline $\mathrm{C}$ & 1.826266000 & -3.665218000 & 0.000000000 \\
\hline $\mathrm{C}$ & 0.528223000 & -3.129743000 & 0.000000000 \\
\hline C & -0.774674000 & -3.924935000 & 0.000000000 \\
\hline $\mathrm{C}$ & -0.475657000 & -5.439377000 & 0.000000000 \\
\hline $\mathrm{C}$ & -1.586655000 & -3.601841000 & 1.274164000 \\
\hline $\mathrm{C}$ & -1.586655000 & -3.601841000 & -1.274164000 \\
\hline $\mathrm{C}$ & -3.842190000 & -0.019191000 & 0.000000000 \\
\hline $\mathrm{C}$ & -4.379653000 & 1.432155000 & 0.000000000 \\
\hline $\mathrm{C}$ & -4.379827000 & -0.744124000 & -1.257571000 \\
\hline $\mathrm{C}$ & -4.379827000 & -0.744124000 & 1.257571000 \\
\hline $\mathrm{H}$ & -4.045887000 & 1.977192000 & 0.885426000 \\
\hline $\mathrm{H}$ & -4.046668000 & -0.247444000 & -2.171301000 \\
\hline $\mathrm{H}$ & -4.043450000 & -1.782581000 & 1.288827000 \\
\hline $\mathrm{H}$ & 3.613844000 & 0.012320000 & 0.000000000 \\
\hline $\mathrm{H}$ & 4.512703000 & 1.857109000 & -3.191126000 \\
\hline $\mathrm{H}$ & 4.612051000 & 1.861570000 & -1.426311000 \\
\hline $\mathrm{H}$ & 4.617582000 & 0.330438000 & -2.306595000 \\
\hline $\mathrm{H}$ & 2.087774000 & 2.364213000 & -4.086660000 \\
\hline $\mathrm{H}$ & -2.552113000 & 0.956589000 & -4.221985000 \\
\hline $\mathrm{H}$ & -1.814329000 & 0.090244000 & -2.861695000 \\
\hline $\mathrm{H}$ & -1.039251000 & 0.058501000 & -4.450224000 \\
\hline $\mathrm{H}$ & -1.822837000 & 2.423058000 & -1.515315000 \\
\hline $\mathrm{H}$ & -2.561402000 & 3.161739000 & -2.948404000 \\
\hline $\mathrm{H}$ & -1.054486000 & 3.818441000 & -2.280499000 \\
\hline
\end{tabular}




\begin{tabular}{|c|c|c|c|}
\hline $\mathrm{H}$ & 0.057257000 & 2.103154000 & -5.418590000 \\
\hline $\mathrm{H}$ & -1.454269000 & 2.988247000 & -5.186681000 \\
\hline $\mathrm{H}$ & 0.051165000 & 3.640743000 & -4.530957000 \\
\hline $\mathrm{H}$ & 4.617582000 & 0.330438000 & 2.306595000 \\
\hline $\mathrm{H}$ & 4.612051000 & 1.861570000 & 1.426311000 \\
\hline $\mathrm{H}$ & 4.512703000 & 1.857109000 & 3.191126000 \\
\hline $\mathrm{H}$ & 2.087774000 & 2.364213000 & 4.086660000 \\
\hline $\mathrm{H}$ & -1.454269000 & 2.988247000 & 5.186681000 \\
\hline $\mathrm{H}$ & 0.057257000 & 2.103154000 & 5.418590000 \\
\hline $\mathrm{H}$ & 0.051165000 & 3.640743000 & 4.530957000 \\
\hline $\mathrm{H}$ & -1.822837000 & 2.423058000 & 1.515315000 \\
\hline $\mathrm{H}$ & -2.561402000 & 3.161739000 & 2.948404000 \\
\hline $\mathrm{H}$ & -1.054486000 & 3.818441000 & 2.280499000 \\
\hline $\mathrm{H}$ & -2.552113000 & 0.956589000 & 4.221985000 \\
\hline $\mathrm{H}$ & -1.814329000 & 0.090244000 & 2.861695000 \\
\hline $\mathrm{H}$ & -1.039251000 & 0.058501000 & 4.450224000 \\
\hline $\mathrm{H}$ & 4.622639000 & -2.134976000 & -0.883061000 \\
\hline $\mathrm{H}$ & 4.529176000 & -3.663352000 & 0.000000000 \\
\hline $\mathrm{H}$ & 4.622639000 & -2.134976000 & 0.883061000 \\
\hline $\mathrm{H}$ & 2.112588000 & -4.706911000 & 0.000000000 \\
\hline $\mathrm{H}$ & 0.087603000 & -5.744267000 & 0.887768000 \\
\hline $\mathrm{H}$ & 0.087603000 & -5.744267000 & -0.887768000 \\
\hline $\mathrm{H}$ & -1.419040000 & -5.996508000 & 0.000000000 \\
\hline $\mathrm{H}$ & -1.809928000 & -2.537702000 & 1.345761000 \\
\hline $\mathrm{H}$ & -2.534300000 & -4.154573000 & 1.273583000 \\
\hline $\mathrm{H}$ & -1.026562000 & -3.890945000 & 2.170675000 \\
\hline $\mathrm{H}$ & -1.026562000 & -3.890945000 & -2.170675000 \\
\hline $\mathrm{H}$ & -2.534300000 & -4.154573000 & -1.273583000 \\
\hline $\mathrm{H}$ & -1.809928000 & -2.537702000 & -1.345761000 \\
\hline $\mathrm{H}$ & -5.476581000 & 1.425495000 & 0.000000000 \\
\hline $\mathrm{H}$ & -4.045887000 & 1.977192000 & -0.885426000 \\
\hline $\mathrm{H}$ & -4.043450000 & -1.782581000 & -1.288827000 \\
\hline $\mathrm{H}$ & -5.476708000 & -0.742942000 & -1.251777000 \\
\hline $\mathrm{H}$ & -5.476708000 & -0.742942000 & 1.251777000 \\
\hline $\mathrm{H}$ & -4.046668000 & -0.247444000 & 2.171301000 \\
\hline \multicolumn{4}{|c|}{$\mathrm{Cr}^{\mathrm{III}}\left(\mathrm{Tp}^{t \mathrm{Bu}, \mathrm{Me}}\right)\left(\mathrm{N}^{t} \mathrm{Bu}\right), \mathrm{S}=3 / 2, \mathrm{C}_{\mathrm{S}}, \mathrm{OLYP}$} \\
\hline $\mathrm{Cr}$ & -0.745310000 & -0.005900000 & 0.000000000 \\
\hline $\mathrm{N}$ & 1.945719000 & 0.744427000 & -1.275331000 \\
\hline $\mathrm{N}$ & 0.614134000 & 0.897758000 & -1.550245000 \\
\hline $\mathrm{N}$ & 1.945719000 & 0.744427000 & 1.275331000 \\
\hline $\mathrm{N}$ & 0.614134000 & 0.897758000 & 1.550245000 \\
\hline $\mathrm{N}$ & 1.953182000 & -1.463506000 & 0.000000000 \\
\hline $\mathrm{N}$ & 0.623329000 & -1.786111000 & 0.000000000 \\
\hline $\mathrm{N}$ & -2.452584000 & -0.009905000 & 0.000000000 \\
\hline B & 2.428161000 & 0.010415000 & 0.000000000 \\
\hline $\mathrm{C}$ & 4.199719000 & 1.332089000 & -2.280354000 \\
\hline $\mathrm{C}$ & 2.701714000 & 1.310203000 & -2.251179000 \\
\hline C & 1.829165000 & 1.846372000 & -3.186222000 \\
\hline $\mathrm{C}$ & 0.531850000 & 1.573570000 & -2.722046000 \\
\hline $\mathrm{C}$ & -0.776791000 & 1.962472000 & -3.404877000 \\
\hline $\mathrm{C}$ & -1.583120000 & 0.691859000 & -3.755694000 \\
\hline $\mathrm{C}$ & -1.592340000 & 2.896711000 & -2.483013000 \\
\hline $\mathrm{C}$ & -0.488543000 & 2.721537000 & -4.717622000 \\
\hline $\mathrm{C}$ & 4.199719000 & 1.332089000 & 2.280354000 \\
\hline
\end{tabular}




\begin{tabular}{|c|c|c|c|}
\hline C & 2.701714000 & 1.310203000 & 2.251179000 \\
\hline C & 1.829165000 & 1.846372000 & 3.186222000 \\
\hline C & 0.531850000 & 1.573570000 & 2.722046000 \\
\hline $\mathrm{C}$ & -0.776791000 & 1.962472000 & 3.404877000 \\
\hline$c$ & -0.488543000 & 2.721537000 & 4.717622000 \\
\hline C & -1.592340000 & 2.896711000 & 2.483013000 \\
\hline $\mathrm{c}$ & -1.583120000 & 0.691859000 & 3.755694000 \\
\hline$c$ & 4.213854000 & -2.614103000 & 0.000000000 \\
\hline 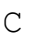 & 2.715670000 & -2.586950000 & 0.000000000 \\
\hline 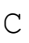 & 1.849551000 & -3.669975000 & 0.000000000 \\
\hline$c$ & 0.549042000 & -3.139461000 & 0.00000000 \\
\hline$c$ & -0.754504000 & -3.933593000 & 0.000000000 \\
\hline$c$ & -0.456241000 & -5.448075000 & 0.00000000 \\
\hline C & -1.567619000 & -3.607669000 & 1.27290700 \\
\hline$c$ & -1.567619000 & -3.607669000 & -1.27290700 \\
\hline c & -3.907114000 & -0.014829000 & 0.00000000 \\
\hline$c$ & -4.447445000 & 1.437065000 & 0.00000000 \\
\hline$c$ & -4.439019000 & -0.743629000 & -1.25921800 \\
\hline$c$ & -4.439019000 & -0.743629000 & 1.25921800 \\
\hline $\mathrm{H}$ & -4.111160000 & 1.981445000 & 0.88547900 \\
\hline $\mathrm{H}$ & -4.102535000 & -0.246564000 & -2.17203100 \\
\hline $\mathrm{H}$ & -4.096176000 & -1.780532000 & 1.28706700 \\
\hline $\mathrm{H}$ & 3.617693000 & 0.014238000 & 0.00000000 \\
\hline $\mathrm{H}$ & 4.531709000 & 1.852285000 & -3.18255200 \\
\hline F & 4.624149000 & 1.855818000 & -1.41758400 \\
\hline $\mathrm{H}$ & 4.629201000 & 0.325116000 & -2.298359000 \\
\hline $\mathrm{H}$ & 2.115250000 & 2.367517000 & -4.08841300 \\
\hline H & -2.532025000 & 0.961540000 & -4.23575100 \\
\hline $\mathrm{H}$ & -1.805610000 & 0.101821000 & -2.86648300 \\
\hline $\mathrm{H}$ & -1.020156000 & 0.057677000 & -4.44985100 \\
\hline $\mathrm{H}$ & -1.816759000 & 2.419938000 & -1.528770000 \\
\hline $\mathrm{H}$ & -2.540491000 & 3.172798000 & -2.960916000 \\
\hline $\mathrm{H}$ & -1.035249000 & 3.817830000 & -2.27774200 \\
\hline H & 0.076571000 & 2.108730000 & -5.42727000 \\
\hline $\mathrm{H}$ & -1.435039000 & 2.994479000 & -5.19723900 \\
\hline $\mathrm{H}$ & 0.070115000 & 3.646057000 & -4.53970800 \\
\hline $\mathrm{H}$ & 4.629201000 & 0.325116000 & 2.298359000 \\
\hline $\mathrm{H}$ & 4.624149000 & 1.855818000 & 1.417584000 \\
\hline $\mathrm{H}$ & 4.531709000 & 1.852285000 & 3.18255200 \\
\hline $\mathrm{H}$ & 2.115250000 & 2.367517000 & 4.08841300 \\
\hline $\mathrm{H}$ & -1.435039000 & 2.994479000 & 5.19723900 \\
\hline $\mathrm{H}$ & 0.076571000 & 2.108730000 & 5.42727000 \\
\hline $\mathrm{H}$ & 0.070115000 & 3.646057000 & 4.539708000 \\
\hline $\mathrm{H}$ & -1.816759000 & 2.419938000 & 1.528770000 \\
\hline $\mathrm{H}$ & -2.540491000 & 3.172798000 & 2.96091600 \\
\hline $\mathrm{H}$ & -1.035249000 & 3.817830000 & 2.27774200 \\
\hline $\mathrm{H}$ & -2.532025000 & 0.961540000 & 4.23575100 \\
\hline $\mathrm{H}$ & -1.805610000 & 0.101821000 & 2.866483000 \\
\hline $\mathrm{H}$ & -1.020156000 & 0.057677000 & 4.449851000 \\
\hline $\mathrm{H}$ & 4.637779000 & -2.124865000 & -0.88300000 \\
\hline H & 4.552141000 & -3.653494000 & 0.00000000 \\
\hline $\mathrm{H}$ & 4.637779000 & -2.124865000 & 0.88300000 \\
\hline $\mathrm{H}$ & 2.141895000 & -4.710103000 & 0.00000000 \\
\hline $\mathrm{H}$ & 0.107654000 & -5.752511000 & 0.887585000 \\
\hline $\mathrm{H}$ & 0.107654000 & -5.752511000 & -0.887585000 \\
\hline 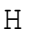 & -1.399119000 & -6.006062000 & 0.00000000 \\
\hline $\mathrm{H}$ & -1.798541000 & -2.544319000 & 1.33809100 \\
\hline
\end{tabular}




$\begin{array}{ll}\mathrm{H} & -2.512275000 \\ \mathrm{H} & -1.005597000 \\ \mathrm{H} & -1.005597000 \\ \mathrm{H} & -2.512275000 \\ \mathrm{H} & -1.798541000 \\ \mathrm{H} & -5.544345000 \\ \mathrm{H} & -4.111160000 \\ \mathrm{H} & -4.096176000 \\ \mathrm{H} & -5.535878000 \\ \mathrm{H} & -5.535878000 \\ \mathrm{H} & -4.102535000\end{array}$

-4.165508000
-3.887053000

$-3.887053000$

$-4.165508000$

$-2.544319000$

1.432164000

1.981445000

$-1.780532000$

$-0.747820000$

$-0.747820000$

$-0.246564000$
1.276303000
2.171252000
$-2.171252000$
$-1.276303000$
$-1.338091000$
0.000000000
$-0.885479000$
$-1.287067000$
$-1.258671000$
1.258671000
2.172031000

$\begin{array}{lrrr}\mathrm{Mn}^{\mathrm{III}}\left(\mathrm{TP}^{t \mathrm{Bu}, \mathrm{Me}}\right)\left(\mathrm{N}^{t} \mathrm{Bu}\right), \mathrm{S}=\mathrm{N}, \mathrm{C}_{3 \mathrm{~V}}, \mathrm{OLYP} & \\ \mathrm{Mn} & 0.000000000 & 0.000000000 & -0.075626000 \\ \mathrm{~N} & 0.729965000 & -1.264336000 & 2.500316000 \\ \mathrm{~N} & 0.870165000 & -1.507170000 & 1.159918000 \\ \mathrm{~N} & 0.729965000 & 1.264336000 & 2.500316000 \\ \mathrm{~N} & 0.870165000 & 1.507170000 & 1.159918000 \\ \mathrm{~N} & -1.459930000 & 0.00000000 & 2.500316000 \\ \mathrm{~N} & -1.740330000 & 0.000000000 & 1.159918000 \\ \mathrm{~N} & 0.000000000 & 0.000000000 & -1.731912000 \\ \mathrm{~B} & 0.000000000 & 0.000000000 & 2.996526000 \\ \mathrm{C} & 1.331022000 & -2.305399000 & 4.729446000 \\ \mathrm{C} & 1.300907000 & -2.253236000 & 3.232981000 \\ \mathrm{C} & 1.829573000 & -3.168914000 & 2.336906000 \\ \mathrm{C} & 1.548685000 & -2.682401000 & 1.050110000 \\ \mathrm{C} & 1.937666000 & -3.356136000 & -0.261446000 \\ \mathrm{C} & 0.665030000 & -3.704779000 & -1.063851000 \\ \mathrm{C} & 2.875918000 & -2.428322000 & -1.063851000 \\ \mathrm{C} & 2.697500000 & -4.672207000 & 0.012189000 \\ \mathrm{C} & 1.331022000 & 2.305399000 & 4.729446000 \\ \mathrm{C} & 1.300907000 & 2.253236000 & 3.232981000 \\ \mathrm{C} & 1.829573000 & 3.168914000 & 2.336906000 \\ \mathrm{C} & 1.548685000 & 2.682401000 & 1.050110000 \\ \mathrm{C} & 1.937666000 & 3.356136000 & -0.261446000 \\ \mathrm{C} & 2.697500000 & 4.672207000 & 0.012189000 \\ \mathrm{C} & 2.875918000 & 2.428322000 & -1.063851000 \\ \mathrm{C} & 0.665030000 & 3.704779000 & -1.063851000 \\ \mathrm{C} & -2.662045000 & 0.000000000 & 4.729446000 \\ \mathrm{C} & -2.601813000 & 0.000000000 & 3.232981000 \\ \mathrm{C} & -3.659147000 & 0.000000000 & 2.336906000 \\ \mathrm{C} & -3.097370000 & 0.000000000 & 1.050110000 \\ \mathrm{C} & -3.875332000 & 0.000000000 & -0.261446000 \\ \mathrm{C} & -5.394999000 & 0.000000000 & 0.012189000 \\ \mathrm{C} & -3.540948000 & 1.276457000 & -1.063851000 \\ \mathrm{C} & -3.540948000 & -1.276457000 & -1.063851000 \\ \mathrm{C} & 0.000000000 & 0.000000000 & -3.203829000 \\ \mathrm{C} & 1.449787000 & 0.000000000 & -3.745733000 \\ \mathrm{C} & -0.724894000 & -1.255553000 & -3.745733000 \\ \mathrm{C} & -0.724894000 & 1.255553000 & -3.745733000 \\ \mathrm{H} & 1.996873000 & 0.885318000 & -3.418206000 \\ \mathrm{H} & -0.231729000 & -2.172002000 & -3.418206000 \\ \mathrm{H} & -1.765144000 & 1.286684000 & -3.418206000 \\ \mathrm{H} & 0.000000000 & 0.000000000 & 4.186893000 \\ \mathrm{H} & 1.854227000 & -3.211616000 & 5.045312000\end{array}$




\begin{tabular}{|c|c|c|c|}
\hline $\mathrm{H}$ & 1.855418000 & -1.447723000 & 5.163943000 \\
\hline $\mathrm{H}$ & 0.326056000 & -2.330700000 & 5.163943000 \\
\hline $\mathrm{H}$ & 2.353529000 & -4.076433000 & 2.599075000 \\
\hline $\mathrm{H}$ & 0.930219000 & -4.155533000 & -2.028197000 \\
\hline $\mathrm{H}$ & 0.058870000 & -2.819059000 & -1.248565000 \\
\hline $\mathrm{H}$ & 0.049648000 & -4.424067000 & -0.511617000 \\
\hline $\mathrm{H}$ & 2.411942000 & -1.460512000 & -1.248565000 \\
\hline $\mathrm{H}$ & 3.133688000 & -2.883360000 & -2.028197000 \\
\hline $\mathrm{H}$ & 3.806531000 & -2.255031000 & -0.511617000 \\
\hline $\mathrm{H}$ & 2.086425000 & -5.389667000 & 0.569188000 \\
\hline $\mathrm{H}$ & 2.967148000 & -5.139251000 & -0.941688000 \\
\hline $\mathrm{H}$ & 3.624376000 & -4.501730000 & 0.569188000 \\
\hline $\mathrm{H}$ & 0.326056000 & 2.330700000 & 5.163943000 \\
\hline $\mathrm{H}$ & 1.855418000 & 1.447723000 & 5.163943000 \\
\hline $\mathrm{H}$ & 1.854227000 & 3.211616000 & 5.045312000 \\
\hline $\mathrm{H}$ & 2.353529000 & 4.076433000 & 2.599075000 \\
\hline $\mathrm{H}$ & 2.967148000 & 5.139251000 & -0.941688000 \\
\hline $\mathrm{H}$ & 2.086425000 & 5.389667000 & 0.569188000 \\
\hline $\mathrm{H}$ & 3.624376000 & 4.501730000 & 0.569188000 \\
\hline $\mathrm{H}$ & 2.411942000 & 1.460512000 & -1.248565000 \\
\hline $\mathrm{H}$ & 3.133688000 & 2.883360000 & -2.028197000 \\
\hline $\mathrm{H}$ & 3.806531000 & 2.255031000 & -0.511617000 \\
\hline $\mathrm{H}$ & 0.930219000 & 4.155533000 & -2.028197000 \\
\hline $\mathrm{H}$ & 0.058870000 & 2.819059000 & -1.248565000 \\
\hline $\mathrm{H}$ & 0.049648000 & 4.424067000 & -0.511617000 \\
\hline $\mathrm{H}$ & -2.181474000 & -0.882977000 & 5.163943000 \\
\hline $\mathrm{H}$ & -3.708454000 & 0.000000000 & 5.045312000 \\
\hline $\mathrm{H}$ & -2.181474000 & 0.882977000 & 5.163943000 \\
\hline $\mathrm{H}$ & -4.707059000 & 0.000000000 & 2.599075000 \\
\hline $\mathrm{H}$ & -5.710801000 & 0.887936000 & 0.569188000 \\
\hline $\mathrm{H}$ & -5.710801000 & -0.887936000 & 0.569188000 \\
\hline $\mathrm{H}$ & -5.934296000 & 0.000000000 & -0.941688000 \\
\hline $\mathrm{H}$ & -2.470812000 & 1.358547000 & -1.248565000 \\
\hline $\mathrm{H}$ & -4.063907000 & 1.272173000 & -2.028197000 \\
\hline $\mathrm{H}$ & -3.856179000 & 2.169037000 & -0.511617000 \\
\hline $\mathrm{H}$ & -3.856179000 & -2.169037000 & -0.511617000 \\
\hline $\mathrm{H}$ & -4.063907000 & -1.272173000 & -2.028197000 \\
\hline $\mathrm{H}$ & -2.470812000 & -1.358547000 & -1.248565000 \\
\hline $\mathrm{H}$ & 1.431768000 & 0.000000000 & -4.842346000 \\
\hline $\mathrm{H}$ & 1.996873000 & -0.885318000 & -3.418206000 \\
\hline $\mathrm{H}$ & -1.765144000 & -1.286684000 & -3.418206000 \\
\hline $\mathrm{H}$ & -0.715884000 & -1.239947000 & -4.842346000 \\
\hline $\mathrm{H}$ & -0.715884000 & 1.239947000 & -4.842346000 \\
\hline $\mathrm{H}$ & -0.231729000 & 2.172002000 & -3.418206000 \\
\hline \multicolumn{4}{|c|}{$\mathrm{Mn}^{\mathrm{III}}\left(\mathrm{Tp}^{t \mathrm{Bu}, \mathrm{Me}}\right)\left(\mathrm{N}^{t} \mathrm{Bu}\right), \mathrm{S}=1, \mathrm{C}_{\mathrm{S}}, \mathrm{OLYP}$} \\
\hline $\mathrm{Mn}$ & -0.666875000 & -0.022429000 & 0.000000000 \\
\hline $\mathrm{N}$ & 1.929111000 & 0.739063000 & -1.266185000 \\
\hline $\mathrm{N}$ & 0.592273000 & 0.893500000 & -1.518125000 \\
\hline $\mathrm{N}$ & 1.929111000 & 0.739063000 & 1.266185000 \\
\hline $\mathrm{N}$ & 0.592273000 & 0.893500000 & 1.518125000 \\
\hline $\mathrm{N}$ & 1.949847000 & -1.468548000 & 0.000000000 \\
\hline $\mathrm{N}$ & 0.619483000 & -1.783394000 & 0.000000000 \\
\hline $\mathrm{N}$ & -2.360387000 & -0.002326000 & 0.000000000 \\
\hline B & 2.423624000 & 0.009477000 & 0.000000000 \\
\hline
\end{tabular}




\begin{tabular}{|c|c|c|c|}
\hline C & 4.170659000 & 1.324030000 & -2.297156000 \\
\hline C & 2.673744000 & 1.305749000 & -2.252149000 \\
\hline C & 1.788627000 & 1.845078000 & -3.170176000 \\
\hline C & 0.495437000 & 1.571679000 & -2.691623000 \\
\hline C & -0.811513000 & 1.965532000 & -3.374496000 \\
\hline C & -1.617323000 & 0.697140000 & -3.733344000 \\
\hline C & -1.624188000 & 2.904941000 & -2.45639400 \\
\hline C & -0.522506000 & 2.725634000 & -4.687260000 \\
\hline C & 4.170659000 & 1.324030000 & 2.29715600 \\
\hline C & 2.673744000 & 1.305749000 & 2.25214900 \\
\hline C & 1.788627000 & 1.845078000 & 3.170176000 \\
\hline C & 0.495437000 & 1.571679000 & 2.691623000 \\
\hline C & -0.811513000 & 1.965532000 & 3.37449600 \\
\hline C & -0.522506000 & 2.725634000 & 4.68726000 \\
\hline C & -1.624188000 & 2.904941000 & 2.45639400 \\
\hline C & -1.617323000 & 0.697140000 & 3.73334400 \\
\hline C & 4.202548000 & -2.626123000 & 0.00000000 \\
\hline C & 2.704823000 & -2.594784000 & 0.00000000 \\
\hline C & 1.832085000 & -3.673671000 & 0.00000000 \\
\hline C & 0.534726000 & -3.137692000 & 0.00000000 \\
\hline C & -0.771181000 & -3.927552000 & 0.00000000 \\
\hline C & -0.479073000 & -5.443318000 & 0.00000000 \\
\hline C & -1.581954000 & -3.600572000 & 1.27391700 \\
\hline C & -1.581954000 & -3.600572000 & -1.27391700 \\
\hline C & -3.816489000 & 0.000318000 & 0.00000000 \\
\hline C & -4.364300000 & 1.450757000 & 0.00000000 \\
\hline C & -4.357009000 & -0.727609000 & -1.257489000 \\
\hline C & -4.357009000 & -0.727609000 & 1.257489000 \\
\hline $\mathrm{H}$ & -4.036686000 & 1.998104000 & 0.88568800 \\
\hline $\mathrm{H}$ & -4.030004000 & -0.231930000 & -2.17332000 \\
\hline $\mathrm{H}$ & -4.022475000 & -1.766383000 & 1.28808100 \\
\hline $\mathrm{H}$ & 3.613154000 & 0.014913000 & 0.00000000 \\
\hline $\mathrm{H}$ & 4.493762000 & 1.847226000 & -3.20090900 \\
\hline $\mathrm{H}$ & 4.605445000 & 1.844181000 & -1.436742000 \\
\hline $\mathrm{H}$ & 4.599448000 & 0.316607000 & -2.32328000 \\
\hline $\mathrm{H}$ & 2.061322000 & 2.369798000 & -4.07427200 \\
\hline $\mathrm{H}$ & -2.573047000 & 0.967934000 & -4.19938100 \\
\hline $\mathrm{H}$ & -1.821655000 & 0.092281000 & -2.85061800 \\
\hline $\mathrm{H}$ & -1.058633000 & 0.076973000 & -4.443448000 \\
\hline $\mathrm{H}$ & -1.829562000 & 2.442158000 & -1.49200200 \\
\hline $\mathrm{H}$ & -2.579956000 & 3.168908000 & -2.92634900 \\
\hline $\mathrm{H}$ & -1.070848000 & 3.832569000 & -2.27137800 \\
\hline $\mathrm{H}$ & 0.041024000 & 2.112959000 & -5.39810400 \\
\hline $\mathrm{H}$ & -1.469476000 & 2.999491000 & -5.16579000 \\
\hline $\mathrm{H}$ & 0.036769000 & 3.649690000 & -4.50950400 \\
\hline $\mathrm{H}$ & 4.599448000 & 0.316607000 & 2.32328000 \\
\hline $\mathrm{H}$ & 4.605445000 & 1.844181000 & 1.43674200 \\
\hline $\mathrm{H}$ & 4.493762000 & 1.847226000 & 3.20090900 \\
\hline $\mathrm{H}$ & 2.061322000 & 2.369798000 & 4.07427200 \\
\hline $\mathrm{H}$ & -1.469476000 & 2.999491000 & 5.165790000 \\
\hline $\mathrm{H}$ & 0.041024000 & 2.112959000 & 5.398104000 \\
\hline $\mathrm{H}$ & 0.036769000 & 3.649690000 & 4.50950400 \\
\hline $\mathrm{H}$ & -1.829562000 & 2.442158000 & 1.49200200 \\
\hline $\mathrm{H}$ & -2.579956000 & 3.168908000 & 2.92634900 \\
\hline $\mathrm{H}$ & -1.070848000 & 3.832569000 & 2.27137800 \\
\hline $\mathrm{H}$ & -2.573047000 & 0.967934000 & 4.199381000 \\
\hline $\mathrm{H}$ & -1.821655000 & 0.092281000 & 2.85061800 \\
\hline
\end{tabular}




$\begin{array}{lr}\mathrm{H} & -1.058633000 \\ \mathrm{H} & 4.626328000 \\ \mathrm{H} & 4.538730000 \\ \mathrm{H} & 4.626328000 \\ \mathrm{H} & 2.117944000 \\ \mathrm{H} & 0.083127000 \\ \mathrm{H} & 0.083127000 \\ \mathrm{H} & -1.424620000 \\ \mathrm{H} & -1.798081000 \\ \mathrm{H} & -2.532947000 \\ \mathrm{H} & -1.024178000 \\ \mathrm{H} & -1.024178000 \\ \mathrm{H} & -2.532947000 \\ \mathrm{H} & -1.798081000 \\ \mathrm{H} & -5.460858000 \\ \mathrm{H} & -4.036686000 \\ \mathrm{H} & -4.022475000 \\ \mathrm{H} & -5.453535000 \\ \mathrm{H} & -5.453535000 \\ \mathrm{H} & -4.030004000 \\ & \end{array}$

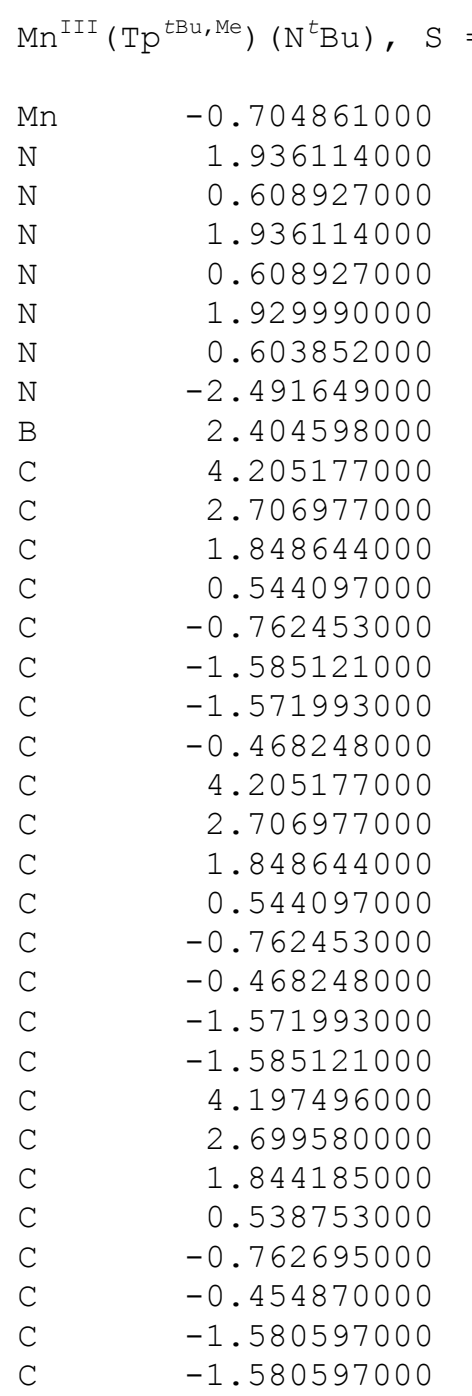

0.076973000

$-2.136850000$

$-3.666092000$

$-2.136850000$

$-4.715530000$

$-5.750279000$

$-5.750279000$

$-5.996883000$

$-2.535352000$

$-4.147628000$

$-3.892927000$

$-3.892927000$

$-4.147628000$

$-2.535352000$

1.430998000

1.998104000

$-1.766383000$

$-0.724341000$

$-0.724341000$

$-0.231930000$
4.443448000
$-0.882921000$
0.000000000
0.882921000
0.000000000
0.887667000
$-0.887667000$
0.000000000
1.347777000
1.272996000
2.170680000
$-2.170680000$
$-1.272996000$
$-1.347777000$
0.000000000
$-0.885688000$
$-1.288081000$
$-1.242654000$
1.242654000
2.173320000

0.000000000

$-0.078624000$

0.764917000

0.932869000

0.764917000

0.932869000

$-1.457791000$

$-1.802268000$

$-0.042401000$

0.028265000

1.331671000

1.321447000

1.863613000

1.603963000

1.997028000

0.731772000

2.943904000

2.740798000

1.331671000

1.321447000

1.863613000

1.603963000

1.997028000

2.740798000

2.943904000

0.731772000

$-2.593619000$

$-2.575990000$

$-3.669471000$

$-3.155560000$

$-3.954727000$

$-5.467227000$

$-3.634221000$

$-3.634221000$
$-1.283076000$

$-1.574549000$

1.283076000

1.574549000

0.000000000

0.000000000

0.000000000

0.000000000

$-2.270789000$

$-2.254797000$

$-3.200199000$

$-2.746894000$

$-3.432807000$

$-3.766237000$

$-2.517975000$

$-4.752439000$

2.270789000

2.254797000

3.200199000

2.746894000

3.432807000

4.752439000

2.517975000

3.766237000

0.000000000

0.000000000

0.000000000

0.000000000

0.000000000

0.000000000

1.271944000

$-1.271944000$ 


\begin{tabular}{|c|c|c|c|}
\hline $\mathrm{C}$ & -3.922650000 & -0.061255000 & 0.000000000 \\
\hline 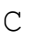 & -4.423594000 & 1.412058000 & 0.000000000 \\
\hline 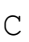 & -4.502389000 & -0.763356000 & -1.262362000 \\
\hline C & -4.502389000 & -0.763356000 & 1.262362000 \\
\hline $\mathrm{H}$ & -4.075542000 & 1.945498000 & 0.887201000 \\
\hline & -4.161454000 & -0.265896000 & -2.173625000 \\
\hline & -4.182752000 & -1.808274000 & 1.30449600 \\
\hline $\mathrm{H}$ & 3.593431000 & 0.018820000 & 0.000000000 \\
\hline $\mathrm{H}$ & 4.548989000 & 1.848938000 & -3.170114000 \\
\hline $\mathrm{H}$ & 4.625806000 & 1.852710000 & -1.404603000 \\
\hline $\mathrm{t}$ & 4.627365000 & 0.321552000 & -2.28504200 \\
\hline & 2.149230000 & 2.379758000 & -4.10084600 \\
\hline & -2.519023000 & 1.007842000 & -4.271656000 \\
\hline $\mathrm{H}$ & -1.840982000 & 0.173248000 & -2.86535000 \\
\hline & -1.022727000 & 0.068312000 & -4.43308200 \\
\hline & -1.806433000 & 2.472339000 & -1.56359100 \\
\hline t & -2.516012000 & 3.225413000 & -3.00094600 \\
\hline $\mathrm{t}$ & -1.008713000 & 3.861303000 & -2.31334400 \\
\hline t & 0.090776000 & 2.115796000 & -5.456367000 \\
\hline H & -1.412063000 & 3.018582000 & -5.234513000 \\
\hline $\mathrm{H}$ & 0.099643000 & 3.661461000 & -4.58354300 \\
\hline & 4.627365000 & 0.321552000 & 2.28504200 \\
\hline $\mathrm{H}$ & 4.625806000 & 1.852710000 & 1.40460300 \\
\hline $\mathrm{H}$ & 4.548989000 & 1.848938000 & 3.17011400 \\
\hline & 2.149230000 & 2.379758000 & 4.100846000 \\
\hline & -1.412063000 & 3.018582000 & 5.234513000 \\
\hline $\mathrm{H}$ & 0.090776000 & 2.115796000 & 5.45636700 \\
\hline & 0.099643000 & 3.661461000 & 4.58354300 \\
\hline $\mathrm{H}$ & -1.806433000 & 2.472339000 & 1.56359100 \\
\hline $\mathrm{H}$ & -2.516012000 & 3.225413000 & 3.00094600 \\
\hline $\mathrm{H}$ & -1.008713000 & 3.861303000 & 2.31334400 \\
\hline $\mathrm{H}$ & -2.519023000 & 1.007842000 & 4.27165600 \\
\hline $\mathrm{H}$ & -1.840982000 & 0.173248000 & 2.86535000 \\
\hline $\mathrm{F}$ & -1.022727000 & 0.068312000 & 4.43308200 \\
\hline $\mathrm{H}$ & 4.617019000 & -2.101402000 & -0.88304800 \\
\hline $\mathrm{H}$ & 4.541782000 & -3.630870000 & 0.000000000 \\
\hline $\mathrm{I}$ & 4.617019000 & -2.101402000 & 0.883048000 \\
\hline $\mathrm{H}$ & 2.148031000 & -4.706242000 & 0.00000000 \\
\hline H & 0.110619000 & -5.768117000 & 0.88771300 \\
\hline $\mathrm{H}$ & 0.110619000 & -5.768117000 & -0.88771300 \\
\hline I & -1.394230000 & -6.030771000 & 0.00000000 \\
\hline $\mathrm{H}$ & -1.835964000 & -2.575897000 & 1.333647000 \\
\hline $\mathrm{H}$ & -2.514793000 & -4.208942000 & 1.276884000 \\
\hline $\mathrm{H}$ & -1.015204000 & -3.898654000 & 2.17256700 \\
\hline $\mathrm{H}$ & -1.015204000 & -3.898654000 & -2.17256700 \\
\hline $\mathrm{H}$ & -2.514793000 & -4.208942000 & -1.27688400 \\
\hline F & -1.835964000 & -2.575897000 & -1.33364700 \\
\hline $\mathrm{F}$ & -5.520000000 & 1.422605000 & 0.000000000 \\
\hline 1 & -4.075542000 & 1.945498000 & -0.887201000 \\
\hline $\mathrm{H}$ & -4.182752000 & -1.808274000 & -1.30449600 \\
\hline & -5.600238000 & -0.749359000 & -1.25198100 \\
\hline & -5.600238000 & -0.749359000 & 1.25198100 \\
\hline & -4.161454000 & -0.265896000 & 2.17362500 \\
\hline
\end{tabular}




\begin{tabular}{|c|c|c|c|}
\hline $\mathrm{Fe}$ & -0.698647000 & -0.042498000 & 0.000000000 \\
\hline $\mathrm{N}$ & 1.943950000 & 0.743820000 & -1.262099000 \\
\hline $\mathrm{N}$ & 0.607770000 & 0.883749000 & -1.506851000 \\
\hline $\mathrm{N}$ & 1.943950000 & 0.743820000 & 1.262099000 \\
\hline $\mathrm{N}$ & 0.607770000 & 0.883749000 & 1.506851000 \\
\hline $\mathrm{N}$ & 1.950646000 & -1.449664000 & 0.000000000 \\
\hline $\mathrm{N}$ & 0.615919000 & -1.745007000 & 0.000000000 \\
\hline $\mathrm{N}$ & -2.363999000 & 0.002283000 & 0.000000000 \\
\hline B & 2.437078000 & 0.016132000 & 0.000000000 \\
\hline C & 4.180297000 & 1.341177000 & -2.294632000 \\
\hline $\mathrm{C}$ & 2.683121000 & 1.311954000 & -2.247825000 \\
\hline $\mathrm{C}$ & 1.791654000 & 1.840304000 & -3.166368000 \\
\hline $\mathrm{C}$ & 0.502322000 & 1.558538000 & -2.681927000 \\
\hline $\mathrm{C}$ & -0.802980000 & 1.950555000 & -3.369625000 \\
\hline $\mathrm{C}$ & -1.606812000 & 0.681816000 & -3.730168000 \\
\hline $\mathrm{C}$ & -1.616628000 & 2.892757000 & -2.455534000 \\
\hline $\mathrm{C}$ & -0.509010000 & 2.708641000 & -4.682924000 \\
\hline $\mathrm{C}$ & 4.180297000 & 1.341177000 & 2.294632000 \\
\hline $\mathrm{C}$ & 2.683121000 & 1.311954000 & 2.247825000 \\
\hline $\mathrm{C}$ & 1.791654000 & 1.840304000 & 3.166368000 \\
\hline $\mathrm{C}$ & 0.502322000 & 1.558538000 & 2.681927000 \\
\hline $\mathrm{C}$ & -0.802980000 & 1.950555000 & 3.369625000 \\
\hline $\mathrm{C}$ & -0.509010000 & 2.708641000 & 4.682924000 \\
\hline $\mathrm{C}$ & -1.616628000 & 2.892757000 & 2.455534000 \\
\hline $\mathrm{C}$ & -1.606812000 & 0.681816000 & 3.730168000 \\
\hline $\mathrm{C}$ & 4.193110000 & -2.625508000 & 0.000000000 \\
\hline $\mathrm{C}$ & 2.695646000 & -2.580144000 & 0.000000000 \\
\hline $\mathrm{C}$ & 1.812616000 & -3.650117000 & 0.000000000 \\
\hline $\mathrm{C}$ & 0.520709000 & -3.102861000 & 0.000000000 \\
\hline $\mathrm{C}$ & -0.780228000 & -3.901120000 & 0.000000000 \\
\hline $\mathrm{C}$ & -0.477510000 & -5.415521000 & 0.000000000 \\
\hline $\mathrm{C}$ & -1.590921000 & -3.583123000 & 1.275810000 \\
\hline $\mathrm{C}$ & -1.590921000 & -3.583123000 & -1.275810000 \\
\hline $\mathrm{C}$ & -3.825020000 & -0.004012000 & 0.000000000 \\
\hline $\mathrm{C}$ & -4.369580000 & 1.446614000 & 0.000000000 \\
\hline $\mathrm{C}$ & -4.354293000 & -0.735044000 & -1.259470000 \\
\hline $\mathrm{C}$ & -4.354293000 & -0.735044000 & 1.259470000 \\
\hline $\mathrm{H}$ & -4.041317000 & 1.993570000 & 0.885682000 \\
\hline $\mathrm{H}$ & -4.025090000 & -0.238310000 & -2.173855000 \\
\hline $\mathrm{H}$ & -4.015271000 & -1.772160000 & 1.287637000 \\
\hline $\mathrm{H}$ & 3.627636000 & 0.015441000 & 0.000000000 \\
\hline $\mathrm{H}$ & 4.499416000 & 1.864713000 & -3.199633000 \\
\hline $\mathrm{H}$ & 4.612154000 & 1.865570000 & -1.435620000 \\
\hline $\mathrm{H}$ & 4.615526000 & 0.336572000 & -2.319092000 \\
\hline $\mathrm{H}$ & 2.058152000 & 2.363538000 & -4.072989000 \\
\hline $\mathrm{H}$ & -2.559928000 & 0.952281000 & -4.201944000 \\
\hline $\mathrm{H}$ & -1.812992000 & 0.078765000 & -2.847015000 \\
\hline $\mathrm{H}$ & -1.044397000 & 0.060931000 & -4.436847000 \\
\hline $\mathrm{H}$ & -1.821441000 & 2.431667000 & -1.490437000 \\
\hline $\mathrm{H}$ & -2.570651000 & 3.157033000 & -2.929060000 \\
\hline $\mathrm{H}$ & -1.062169000 & 3.820249000 & -2.272615000 \\
\hline $\mathrm{H}$ & 0.056229000 & 2.094822000 & -5.391488000 \\
\hline $\mathrm{H}$ & -1.454259000 & 2.982648000 & -5.164671000 \\
\hline $\mathrm{H}$ & 0.050291000 & 3.632653000 & -4.504944000 \\
\hline $\mathrm{H}$ & 4.615526000 & 0.336572000 & 2.319092000 \\
\hline
\end{tabular}




\begin{tabular}{|c|c|c|c|}
\hline $\mathrm{H}$ & 4.612154000 & 1.865570000 & 1.435620000 \\
\hline $\mathrm{H}$ & 4.499416000 & 1.864713000 & 3.199633000 \\
\hline $\mathrm{H}$ & 2.058152000 & 2.363538000 & 4.072989000 \\
\hline $\mathrm{H}$ & -1.454259000 & 2.982648000 & 5.164671000 \\
\hline $\mathrm{H}$ & 0.056229000 & 2.094822000 & 5.391488000 \\
\hline $\mathrm{H}$ & 0.050291000 & 3.632653000 & 4.504944000 \\
\hline $\mathrm{H}$ & -1.821441000 & 2.431667000 & 1.490437000 \\
\hline $\mathrm{H}$ & -2.570651000 & 3.157033000 & 2.929060000 \\
\hline $\mathrm{H}$ & -1.062169000 & 3.820249000 & 2.272615000 \\
\hline $\mathrm{H}$ & -2.559928000 & 0.952281000 & 4.201944000 \\
\hline $\mathrm{H}$ & -1.812992000 & 0.078765000 & 2.847015000 \\
\hline $\mathrm{H}$ & -1.044397000 & 0.060931000 & 4.436847000 \\
\hline $\mathrm{H}$ & 4.621545000 & -2.139960000 & -0.882789000 \\
\hline $\mathrm{H}$ & 4.519983000 & -3.668534000 & 0.000000000 \\
\hline $\mathrm{H}$ & 4.621545000 & -2.139960000 & 0.882789000 \\
\hline $\mathrm{H}$ & 2.089386000 & -4.694161000 & 0.000000000 \\
\hline $\mathrm{H}$ & 0.086210000 & -5.719652000 & 0.887688000 \\
\hline $\mathrm{H}$ & 0.086210000 & -5.719652000 & -0.887688000 \\
\hline $\mathrm{H}$ & -1.419809000 & -5.974607000 & 0.000000000 \\
\hline $\mathrm{H}$ & -1.806943000 & -2.518603000 & 1.354394000 \\
\hline $\mathrm{H}$ & -2.539611000 & -4.134356000 & 1.273716000 \\
\hline $\mathrm{H}$ & -1.030099000 & -3.878841000 & 2.169709000 \\
\hline $\mathrm{H}$ & -1.030099000 & -3.878841000 & -2.169709000 \\
\hline $\mathrm{H}$ & -2.539611000 & -4.134356000 & -1.273716000 \\
\hline $\mathrm{H}$ & -1.806943000 & -2.518603000 & -1.354394000 \\
\hline $\mathrm{H}$ & -5.465890000 & 1.425428000 & 0.000000000 \\
\hline $\mathrm{H}$ & -4.041317000 & 1.993570000 & -0.885682000 \\
\hline $\mathrm{H}$ & -4.015271000 & -1.772160000 & -1.287637000 \\
\hline $\mathrm{H}$ & -5.450718000 & -0.735526000 & -1.248011000 \\
\hline $\mathrm{H}$ & -5.450718000 & -0.735526000 & 1.248011000 \\
\hline $\mathrm{H}$ & -4.025090000 & -0.238310000 & 2.173855000 \\
\hline \multicolumn{4}{|c|}{$\mathrm{Fe}^{\mathrm{III}}\left(\mathrm{Tp}^{t \mathrm{Bu}, \mathrm{Me}}\right)\left(\mathrm{N}^{t} \mathrm{Bu}\right), \mathrm{S}=3 / 2, \mathrm{C}_{\mathrm{S}}, \mathrm{OLYP}$} \\
\hline $\mathrm{Fe}$ & -0.706131000 & -0.126520000 & 0.000000000 \\
\hline $\mathrm{N}$ & 1.925664000 & 0.755294000 & -1.281289000 \\
\hline $\mathrm{N}$ & 0.596557000 & 0.901370000 & -1.573716000 \\
\hline $\mathrm{N}$ & 1.925664000 & 0.755294000 & 1.281289000 \\
\hline $\mathrm{N}$ & 0.596557000 & 0.901370000 & 1.573716000 \\
\hline $\mathrm{N}$ & 1.935007000 & -1.450644000 & 0.000000000 \\
\hline $\mathrm{N}$ & 0.607058000 & -1.780461000 & 0.000000000 \\
\hline $\mathrm{N}$ & -2.436109000 & 0.014702000 & 0.000000000 \\
\hline B & 2.404713000 & 0.032209000 & 0.000000000 \\
\hline $\mathrm{C}$ & 4.191491000 & 1.359818000 & -2.258213000 \\
\hline $\mathrm{C}$ & 2.693208000 & 1.332297000 & -2.245301000 \\
\hline $\mathrm{C}$ & 1.830380000 & 1.868771000 & -3.187171000 \\
\hline $\mathrm{C}$ & 0.527803000 & 1.583940000 & -2.739532000 \\
\hline $\mathrm{C}$ & -0.776127000 & 1.977784000 & -3.428044000 \\
\hline $\mathrm{C}$ & -1.592911000 & 0.712822000 & -3.773755000 \\
\hline $\mathrm{C}$ & -1.584439000 & 2.921334000 & -2.509118000 \\
\hline $\mathrm{C}$ & -0.481315000 & 2.730032000 & -4.743120000 \\
\hline $\mathrm{C}$ & 4.191491000 & 1.359818000 & 2.258213000 \\
\hline $\mathrm{C}$ & 2.693208000 & 1.332297000 & 2.245301000 \\
\hline $\mathrm{C}$ & 1.830380000 & 1.868771000 & 3.187171000 \\
\hline $\mathrm{C}$ & 0.527803000 & 1.583940000 & 2.739532000 \\
\hline $\mathrm{C}$ & -0.776127000 & 1.977784000 & 3.428044000 \\
\hline
\end{tabular}




\begin{tabular}{|c|c|c|c|}
\hline C & -0.481315000 & 2.730032000 & 4.743120000 \\
\hline $\mathrm{C}$ & -1.584439000 & 2.921334000 & 2.509118000 \\
\hline$c$ & -1.592911000 & 0.712822000 & 3.773755000 \\
\hline 0 & 4.195063000 & -2.594510000 & 0.000000000 \\
\hline c & 2.697072000 & -2.570913000 & 0.000000000 \\
\hline$c$ & 1.833354000 & -3.658661000 & 0.000000000 \\
\hline c & 0.532383000 & -3.136099000 & 0.000000000 \\
\hline c & -0.768817000 & -3.932757000 & 0.000000000 \\
\hline$c$ & -0.464542000 & -5.446237000 & 0.00000000 \\
\hline 0 & -1.582668000 & -3.610982000 & 1.27364400 \\
\hline 0 & -1.582668000 & -3.610982000 & -1.27364400 \\
\hline$c$ & -3.869091000 & -0.017058000 & 0.00000000 \\
\hline C & -4.387294000 & 1.450290000 & 0.00000000 \\
\hline c & -4.432637000 & -0.727847000 & -1.26284700 \\
\hline$c$ & -4.432637000 & -0.727847000 & 1.26284700 \\
\hline $\mathrm{H}$ & -4.047224000 & 1.988100000 & 0.88688700 \\
\hline $\mathrm{H}$ & -4.101336000 & -0.225293000 & -2.17410200 \\
\hline $\mathrm{H}$ & -4.100196000 & -1.768037000 & 1.30433200 \\
\hline $\mathrm{H}$ & 3.594104000 & 0.030627000 & 0.00000000 \\
\hline $\mathrm{H}$ & 4.530352000 & 1.883762000 & -3.15577000 \\
\hline $\mathrm{H}$ & 4.606993000 & 1.882501000 & -1.39038900 \\
\hline $\mathrm{H}$ & 4.625805000 & 0.354641000 & -2.27599100 \\
\hline $\mathrm{H}$ & 2.124856000 & 2.397144000 & -4.08268900 \\
\hline $\mathrm{H}$ & -2.535992000 & 0.987612000 & -4.26269400 \\
\hline $\mathrm{H}$ & -1.827715000 & 0.134227000 & -2.88069500 \\
\hline $\mathrm{H}$ & -1.031952000 & 0.067045000 & -4.45894800 \\
\hline $\mathrm{H}$ & -1.795704000 & 2.454440000 & -1.54765800 \\
\hline $\mathrm{H}$ & -2.537107000 & 3.193316000 & -2.98079800 \\
\hline $\mathrm{H}$ & -1.025224000 & 3.844228000 & -2.31822000 \\
\hline $\mathrm{H}$ & 0.082814000 & 2.111880000 & -5.44903300 \\
\hline $\mathrm{H}$ & -1.424996000 & 3.007130000 & -5.22619600 \\
\hline $\mathrm{H}$ & 0.082057000 & 3.652000000 & -4.56689600 \\
\hline $\mathrm{H}$ & 4.625805000 & 0.354641000 & 2.27599100 \\
\hline $\mathrm{H}$ & 4.606993000 & 1.882501000 & 1.390389000 \\
\hline $\mathrm{H}$ & 4.530352000 & 1.883762000 & 3.15577000 \\
\hline $\mathrm{H}$ & 2.124856000 & 2.397144000 & 4.08268900 \\
\hline $\mathrm{H}$ & -1.424996000 & 3.007130000 & 5.22619600 \\
\hline $\mathrm{H}$ & 0.082814000 & 2.111880000 & 5.44903300 \\
\hline $\mathrm{H}$ & 0.082057000 & 3.652000000 & $4.5668960 c$ \\
\hline $\mathrm{H}$ & -1.795704000 & 2.454440000 & 1.54765800 \\
\hline $\mathrm{H}$ & -2.537107000 & 3.193316000 & 2.98079800 \\
\hline $\mathrm{H}$ & -1.025224000 & 3.844228000 & 2.31822000 \\
\hline $\mathrm{H}$ & -2.535992000 & 0.987612000 & 4.26269400 \\
\hline $\mathrm{H}$ & -1.827715000 & 0.134227000 & 2.88069500 \\
\hline $\mathrm{H}$ & -1.031952000 & 0.067045000 & 4.45894800 \\
\hline $\mathrm{H}$ & 4.615758000 & -2.103000000 & -0.88289500 \\
\hline $\mathrm{H}$ & 4.536465000 & -3.632791000 & 0.00000000 \\
\hline $\mathrm{H}$ & 4.615758000 & -2.103000000 & 0.88289500 \\
\hline $\mathrm{H}$ & 2.129423000 & -4.697534000 & 0.00000000 \\
\hline $\mathrm{H}$ & 0.100402000 & -5.748211000 & 0.887636000 \\
\hline $\mathrm{H}$ & 0.100402000 & -5.748211000 & -0.887636000 \\
\hline $\mathrm{H}$ & -1.405218000 & -6.007683000 & 0.00000000 \\
\hline $\mathrm{H}$ & -1.819880000 & -2.549286000 & 1.34260100 \\
\hline $\mathrm{H}$ & -2.524581000 & -4.172944000 & 1.27665800 \\
\hline $\mathrm{H}$ & -1.019240000 & -3.888305000 & 2.17155100 \\
\hline $\mathrm{H}$ & -1.019240000 & -3.888305000 & -2.17155100 \\
\hline $\mathrm{H}$ & -2.524581000 & -4.172944000 & -1.27665800 \\
\hline
\end{tabular}


$-2.549286000$

1. 441803000

1. 988100000

$-1.768037000$

$-0.726007000$

$-0.726007000$

$-0.225293000$
-1.342601000
0.000000000
-0.886887000
-1.304332000
-1.247730000
1.247730000
2.174102000
$F e^{I I I}\left(T p^{t B u, M e}\right)\left(N^{t} B u\right), \quad S=5 / 2, C_{3 V}$, OLYP

\begin{tabular}{|c|c|c|c|}
\hline $\mathrm{Fe}$ & 0.000000000 & 0.000000000 & -0.118905000 \\
\hline $\mathrm{N}$ & 0.740953000 & -1.283368000 & 2.468983000 \\
\hline $\mathrm{N}$ & 0.915674000 & -1.585995000 & 1.142564000 \\
\hline $\mathrm{N}$ & 0.740953000 & 1.283368000 & 2.468983000 \\
\hline $\mathrm{N}$ & 0.915674000 & 1.585995000 & 1.142564000 \\
\hline $\mathrm{N}$ & -1.481905000 & 0.000000000 & 2.468983000 \\
\hline $\mathrm{N}$ & -1.831349000 & 0.000000000 & 1.142564000 \\
\hline $\mathrm{N}$ & 0.000000000 & 0.000000000 & -1.896704000 \\
\hline B & 0.000000000 & 0.000000000 & 2.936421000 \\
\hline $\mathrm{C}$ & 1.302334000 & -2.255709000 & 4.746273000 \\
\hline $\mathrm{C}$ & 1.297528000 & -2.247385000 & 3.247683000 \\
\hline $\mathrm{C}$ & 1.846364000 & -3.197997000 & 2.398859000 \\
\hline $\mathrm{C}$ & 1.592117000 & -2.757628000 & 1.090472000 \\
\hline $\mathrm{C}$ & 1.994254000 & -3.454149000 & -0.205796000 \\
\hline $\mathrm{C}$ & 0.731557000 & -3.809447000 & -1.022774000 \\
\hline $\mathrm{C}$ & 2.933299000 & -2.538271000 & -1.022774000 \\
\hline $\mathrm{C}$ & 2.749794000 & -4.762783000 & 0.107352000 \\
\hline $\mathrm{C}$ & 1.302334000 & 2.255709000 & 4.746273000 \\
\hline $\mathrm{C}$ & 1.297528000 & 2.247385000 & 3.247683000 \\
\hline C & 1.846364000 & 3.197997000 & 2.398859000 \\
\hline $\mathrm{C}$ & 1.592117000 & 2.757628000 & 1.090472000 \\
\hline $\mathrm{C}$ & 1.994254000 & 3.454149000 & -0.205796000 \\
\hline $\mathrm{C}$ & 2.749794000 & 4.762783000 & 0.107352000 \\
\hline $\mathrm{C}$ & 2.933299000 & 2.538271000 & -1.022774000 \\
\hline $\mathrm{C}$ & 0.731557000 & 3.809447000 & -1.022774000 \\
\hline $\mathrm{C}$ & -2.604668000 & 0.000000000 & 4.746273000 \\
\hline $\mathrm{C}$ & -2.595056000 & 0.000000000 & 3.247683000 \\
\hline $\mathrm{C}$ & -3.692729000 & 0.000000000 & 2.398859000 \\
\hline C & -3.184234000 & 0.000000000 & 1.090472000 \\
\hline $\mathrm{C}$ & -3.988508000 & 0.000000000 & -0.205796000 \\
\hline $\mathrm{C}$ & -5.499588000 & 0.000000000 & 0.107352000 \\
\hline $\mathrm{C}$ & -3.664856000 & 1.271176000 & -1.022774000 \\
\hline $\mathrm{C}$ & -3.664856000 & -1.271176000 & -1.022774000 \\
\hline $\mathrm{C}$ & 0.000000000 & 0.000000000 & -3.331179000 \\
\hline $\mathrm{C}$ & 1.452931000 & 0.000000000 & -3.885917000 \\
\hline $\mathrm{C}$ & -0.726466000 & -1.258275000 & -3.885917000 \\
\hline $\mathrm{C}$ & -0.726466000 & 1.258275000 & -3.885917000 \\
\hline $\mathrm{H}$ & 1.997721000 & 0.885552000 & -3.548687000 \\
\hline $\mathrm{H}$ & -0.231950000 & -2.172853000 & -3.548687000 \\
\hline $\mathrm{H}$ & -1.765771000 & 1.287302000 & -3.548687000 \\
\hline $\mathrm{H}$ & 0.000000000 & 0.000000000 & 4.124960000 \\
\hline $\mathrm{H}$ & 1.820057000 & -3.152431000 & 5.096093000 \\
\hline $\mathrm{H}$ & 1.820307000 & -1.386815000 & 5.164656000 \\
\hline $\mathrm{H}$ & 0.290863000 & -2.269839000 & 5.164656000 \\
\hline $\mathrm{H}$ & 2.363896000 & -4.094388000 & 2.709272000 \\
\hline $\mathrm{H}$ & 1.011598000 & -4.313516000 & -1.95584000 \\
\hline
\end{tabular}




$\begin{array}{lrrr}\mathrm{H} & 0.157694000 & -2.918364000 & -1.277696000 \\ \mathrm{H} & 0.080319000 & -4.483767000 & -0.455104000 \\ \mathrm{H} & 2.448531000 & -1.595749000 & -1.277696000 \\ \mathrm{H} & 3.229816000 & -3.032827000 & -1.955840000 \\ \mathrm{H} & 3.842897000 & -2.311442000 & -0.455104000 \\ \mathrm{H} & 2.130472000 & -5.465268000 & 0.674506000 \\ \mathrm{H} & 3.033621000 & -5.254386000 & -0.829506000 \\ \mathrm{H} & 3.667825000 & -4.577677000 & 0.674506000 \\ \mathrm{H} & 0.290863000 & 2.269839000 & 5.164656000 \\ \mathrm{H} & 1.820307000 & 1.386815000 & 5.164656000 \\ \mathrm{H} & 1.820057000 & 3.152431000 & 5.096093000 \\ \mathrm{H} & 2.363896000 & 4.094388000 & 2.709272000 \\ \mathrm{H} & 3.033621000 & 5.254386000 & -0.829506000 \\ \mathrm{H} & 2.130472000 & 5.465268000 & 0.674506000 \\ \mathrm{H} & 3.667825000 & 4.577677000 & 0.674506000 \\ \mathrm{H} & 2.448531000 & 1.595749000 & -1.277696000 \\ \mathrm{H} & 3.229816000 & 3.032827000 & -1.955840000 \\ \mathrm{H} & 3.842897000 & 2.311442000 & -0.455104000 \\ \mathrm{H} & 1.011598000 & 4.313516000 & -1.955840000 \\ \mathrm{H} & 0.157694000 & 2.918364000 & -1.277696000 \\ \mathrm{H} & 0.080319000 & 4.483767000 & -0.455104000 \\ \mathrm{H} & -2.111170000 & -0.883025000 & 5.164656000 \\ \mathrm{H} & -3.640114000 & 0.000000000 & 5.096093000 \\ \mathrm{H} & -2.111170000 & 0.883025000 & 5.164656000 \\ \mathrm{H} & -4.727792000 & 0.000000000 & 2.709272000 \\ \mathrm{H} & -5.798297000 & 0.887591000 & 0.674506000 \\ \mathrm{H} & -5.798297000 & -0.887591000 & 0.674506000 \\ \mathrm{H} & -6.067243000 & 0.000000000 & -0.829506000 \\ \mathrm{H} & -2.606224000 & 1.322615000 & -1.277696000 \\ \mathrm{H} & -4.241413000 & 1.280689000 & -1.955840000 \\ \mathrm{H} & -3.923215000 & 2.172325000 & -0.455104000 \\ \mathrm{H} & -3.923215000 & -2.172325000 & -0.455104000 \\ \mathrm{H} & -4.241413000 & -1.280689000 & -1.955840000 \\ \mathrm{H} & -2.606224000 & -1.322615000 & -1.277696000 \\ \mathrm{H} & 1.449778000 & 0.000000000 & -4.983581000 \\ \mathrm{H} & 1.997721000 & -0.885552000 & -3.548687000 \\ \mathrm{H} & -1.765771000 & -1.287302000 & -3.548687000 \\ \mathrm{H} & -0.724889000 & -1.255544000 & -4.983581000 \\ \mathrm{H} & -0.724889000 & 1.255544000 & -4.983581000 \\ \mathrm{H} & -0.231950000 & 2.172853000 & -3.548687000\end{array}$

